\title{
Wettability and capillary behavior of fibrous gas diffusion media for polymer electrolyte membrane fuel cells
}

Jeff T. Gostick, Marios A. Ioannidis, Michael W. Fowler* and Mark D. Pritzker

Department of Chemical Engineering, University of Waterloo, Waterloo ON N2L 3G1

*Corresponding Author: mfowler@uwaterloo.ca 


\section{Abstract}

The relationship of capillary pressure to liquid saturation for the water-air fluid pair in two different types of gas diffusion media (GDM) used in polymer electrolyte membrane fuel cell (PEMFC) electrodes is elucidated. It is experimentally demonstrated that GDM samples with and without treatment with poly(tetrafluoroethylene) (PTFE) ubiquitously display permanent capillary pressure hysteresis. Water does not imbibe spontaneously into a dry GDM, neither is it ejected spontaneously from a water-saturated GDM. Rather, positive displacement pressure is required to force both water and air into GDMs, whereas the main effect of adding PTFE is to increase the amount of work required for forcing water into the GDM, and to decrease the work required for water removal. Irrespective of PTFE content, the GDM samples tested are generally shown to behave as materials of intermediate (neutral) wettability. The US Bureau of Mines (USBM) wettability index nevertheless shows that water is the preferentially non-wetting phase in PTFE-treated GDMs and the preferentially wetting phase in untreated GDMs. Water-air capillary pressure curves are found to depend on sample thickness, clearly demonstrating that finite-size effects are important. Finally, compression of the GDM is found to increase the capillary pressures for water injection and decrease the capillary pressures required for water withdrawal. These results should aid the design of GDMs with improved water management properties and the modeling of PEMFC electrodes in general. 


\section{Introduction}

Hydrogen figures largely in most visions of a sustainable energy future and the term "hydrogen economy" is virtually synonymous with green energy, renewable resources and sustainability $[1,2]$. The fuel cell is a key component of the hydrogen economy since it converts hydrogen fuel into useful power, with only water and heat as byproducts. Recent advances in fuel cell technology have brought the hydrogen economy vision closer to reality. The development of very thin proton conducting membranes [3] has dramatically improved cell performance by reducing ohmic losses, while development of low platinum loading electrodes has significantly reduced cost [4]. Nonetheless, further improvements are needed before fuel cells will be ready for broad commercialization. One of the main target areas for improvement is overall fuel cell performance.

The performance of the polymer electrolyte membrane fuel cell (PEMFC) can be adversely affected by excessive accumulation of liquid water inside the cell. Its presence in the pores of the cathode gas diffusion medium (GDM) and catalyst layer reduces the effective diffusivity of oxygen to the catalytic sites. The diminished flux of oxygen leads to reduced oxygen concentration at the catalytic sites, which in turn leads to increased concentration polarization and reduced power output. Ultimately, severe water-flooding causes the maximum achievable current density to become limited by reactant mass transfer to the catalytic sites (i.e. limiting current). Maximizing fuel cell performance thus requires effective strategies for preventing the accumulation of water in the porous components of the fuel cell. A common strategy is to impregnate the GDM with a 
hydrophobic polymer, such as poly(tetrafluoroethylene) (PTFE), to render the carbon fibers more hydrophobic. Although this treatment is known to be effective for high humidity and high current density operation, very little is actually known about how PTFE addition alters the wettability and capillary properties of the GDM. Sinha et al. [5] have recently reviewed efforts to understand liquid water transport in PTFE-treated GDMs.

Several attempts have been made to quantify the effect of PTFE coatings on GDM wettability. The contact angle of water on the exterior GDM surface has been measured as a function of hydrophobic polymer content [6,7], but relating this macroscopic measurement to the intrinsic contact angle inside the GDM is not straightforward. The contact angle measured on the surface of a GDM is affected by porosity, macroscopic roughness created by the fibers ridges, microscopic roughness of the individual fibers, and chemical heterogeneity of the carbon and PTFE surfaces. One can attempt to account for these effects using the Wenzel and Cassie-Baxter equations [8], but this requires estimates of the roughness and the ratio of solid-to-pore area on the surface, which are not simple to obtain for anisotropic material like fibrous GDMs [9]. Gurau et al. [10] proposed a method for estimating the internal contact angle of GDMs, but their approach uses highly wetting fluids other than water (e.g., octane) to infer the contact angle of water. Other attempts to determine the wettability of GDMs have included measurements of water uptake [11,12] or water breakthrough pressure [13], but these procedures have provided only limited qualitative information. 
A preferred approach to assessing GDM wettability is to measure the water-air capillary pressure curves directly, since the capillary pressure behavior of the GDM is precisely what the hydrophobic polymer treatment is intended to alter. Early attempts at such a measurement using the method of standard porosimetry (MSP) have been made by Gostick et al. [9] and more recently by others [14-16]. Using this method, the capillary pressure, defined as $P_{C} \equiv P_{L}-P_{G}$, was measured as the water saturation of a GDM sample was gradually decreased. It was found that water withdrawal was associated with negative capillary pressures. This observation was attributed to the presence of a network of hydrophilic pores presumed to be composed of graphite surfaces that were not covered by the PTFE coating. Apparently conflicting evidence was provided by Benziger et al. [13], who showed that positive capillary pressures were required to inject water into a GDM, even in the absence of PTFE coating. Spontaneous imbibition of water, i.e. water uptake by the GDM at $P_{C} \leq 0$, would be expected if a network of hydrophilic pores existed, but this was not observed. More recently, efforts have been made by a number of groups to develop alternative methods for measuring the water-air capillary pressure curves of GDMs [17-23]. Fairweather et al. [18] reported a technique that allowed both injection and withdrawal of water. Their data revealed that GDM capillary pressure curves exhibit large permanent hysteresis, to the extent that water injection and withdrawal occur at positive and negative capillary pressures, respectively. Such extensive capillary pressure hysteresis was confirmed by the measurements of Harkness et al. [21] and Gostick et al. [20]. According to conventional understanding [24], the observed hysteretic behavior corresponds to an apparent switch in wettability, which is consistent $[25,26]$ with the fact that both graphite and PTFE are materials of intermediate 
(neutral) wettability; $\theta \approx 75^{\circ}-86^{\circ}$ for water on graphite $[8,27,28]$ and $\theta \approx 108^{\circ}-112^{\circ}$ for water on PTFE [29,30].

In this work, water injection and withdrawal capillary pressure curves for GDMs with various PTFE loadings are systematically obtained using a previously reported method [20]. The results are analyzed in terms of the US Bureau of Mines (USBM) wettability index [25] which provides a simple and sensitive means of quantitatively describing the effect of a hydrophobic coating on observed behavior. The effects of GDM compression and sample thickness on capillary pressure curves are also investigated. The data obtained support the general conclusion that the wettability of GDM-water-air systems is intermediate (neutral). A view of GDMs as materials of mixed wettability with capillary properties that are sensitive to the relative abundance of hydrophilic pores [5,31-33] is not supported by experiment. 


\section{Experimental}

Water-air capillary pressure curves of GDMs were measured using a method first described elsewhere [20], with a slight modification to the sample holder to facilitate compression of the GDM sample. Mercury capillary pressure curves were also measured on GDM samples to provide a baseline so that pore structure and wettability changes could be differentiated.

\subsection{Water-air Capillary Pressure Measurement}

A distinct advantage of the method used in this work [20] is that capillary pressure (the controlled variable) is increased in a stepwise fashion while water saturation is measured at each step. Straightforward interpretation of the data is thus possible, unlike other approaches in which the pressure response is monitored as water is injected (or withdrawn) in a stepwise [18] or continuous fashion [21]. Interpretation of porosimetry data obtained under volume-controlled conditions is not trivial [34-37]. An analysis of the various experimental techniques that have recently been proposed may be found in Gostick et al. [38].

The experimental setup used here consists of an analytical balance, syringe pump, absolute pressure gauge and specially designed sample holder. The overall system setup is shown in Figure 1. The sample is positioned in the sample holder so that water has access to its bottom face while gas moves in and out through the top. The syringe pump adjusts the gas pressure by advancing and retracting the syringe to expand and compress 
the gas in the sample. Since the liquid pressure remains constant, controlling the gas pressure controls the capillary pressure $\left(P_{C}=P_{L}-P_{G}\right)$. A feedback control algorithm was developed in Labview to control the direction and speed of pump movements so that each capillary pressure set point is achieved and maintained. The GDM saturation was found by monitoring the mass of water in the reservoir on the analytical balance. Since the GDM starts completely dry the amount water in the sample is equal to the amount missing from the reservoir. Converting the mass of water $m_{W}$ in the GDM to saturation $S_{W}$ was done with the following equation:

$$
S_{W}=\frac{V_{W}}{V_{p}}=\frac{m_{W} \cdot \rho^{-1}}{\frac{\pi}{4} d^{2} \delta \cdot \varepsilon}
$$

where $d$ is the sample diameter, $\delta$ is sample thickness, $\varepsilon$ is the sample porosity and $\rho$ is the density of water. After reaching a capillary pressure set point, the mass on the reservoir is monitored for change. When the rate of change of reservoir mass, and therefore GDM saturation, is sufficiently slow the GDM saturation is deemed stable and the next capillary pressure set point is applied. Further details regarding the sample mounting, system setup and experimental procedure are given elsewhere [20].

For the present study, a slight modification to the previously described sample holder [20] was required, as shown in Figure 2. The main modification is the addition of a set screw threaded into the compression cylinder. Once the sample is mounted and the holder is assembled, the set screw is tightened by inserting a hex key in the gas port. For tests on uncompressed samples, the set screw is turned until light contact is made with the plug. Application of firm pressure to the set screw compresses the GDM. This approach is not intended to precisely simulate the compression conditions existing in fuel cells, but rather 
to investigate qualitatively the effect of compression on capillary properties. Uniformity of sample compression or damage sustained by the sample due to compression was not analyzed. Because of the simplistic method used to apply compression, the amount of force exerted on the sample was unknown. The extent of compression cannot be explicitly controlled with this setup, but it can be found after the experiment by analysis of the resulting data. Specifically, the reduction in pore volume can be determined from the data using the following equation:

$$
S_{W}=\frac{V_{W}}{V_{p, C}}=\frac{m_{W} \cdot \rho^{-1}}{\frac{\pi}{4} d^{2} \delta_{C} \cdot \varepsilon_{C}}
$$

where $\delta_{C}$ and $\varepsilon_{C}$ are the thickness and porosity, respectively, of the compressed sample, $d$ is the diameter of the circular sample, $\rho$ is the density of water, $S_{W}$ is the water saturation and $m_{W}$ is the mass of water in the sample. Values of $\delta_{C}$ and $\varepsilon_{C}$ are found that allow the maximum water saturation measured in the experiment to be unity. This assumes that the samples were fully saturated at the maximum pressure attained during the experiment, an assumption confirmed for uncompressed samples for which the thickness and porosity is known. The determination of unique values of $\delta_{C}$ and $\varepsilon_{C}$ from Eq. (2) requires that an additional expression be used. Such an expression can be derived based on the fact that $\varepsilon_{C}$ can be related to $\delta_{C}$ by assuming that (a) the solid does not compress and (b) the sample only deforms in the direction of compression. The latter assumption is reasonable, considering that the sample is laterally confined by the sample locating gasket (see Figure 2). The former assumption is also valid, considering the high sample porosity and rigid nature of graphite fibers, and is independent of possible fiber breakage. Together, these assumptions lead to the following expression for compressed porosity [39]: 


$$
\varepsilon_{C}=1-\frac{\delta_{O}}{\delta_{C}}\left(1-\varepsilon_{O}\right)
$$

where $\delta_{0}$ and $\varepsilon_{0}$ are the thickness and porosity of the uncompressed sample, respectively. This equation can be then be combined with Eq. (2) to obtain a unique value of $\delta_{C}$.

\subsection{Mercury Intrusion Porosimetry}

Mercury intrusion porosimetry (MIP) is well suited to the study of the microstructure of porous materials because mercury is a highly non-wetting fluid on most surfaces. Thus, MIP is used in this study to gauge the effect of PTFE addition on the structure of GDMs so that the effects of changes in wettability and pore microstructure on the water-air capillary pressure curves can be differentiated and the proper interpretations of the experimental data made. A Quantachrome Poremaster was used for MIP testing with triply distilled ACS grade mercury (99.99\% purity). A single strip of GDM, about $1 \mathrm{~cm} \times$ $3 \mathrm{~cm}$, was analyzed in each MIP test of this study.

\subsection{GDM Materials}

Toray and SGL10 series materials with varying amounts of PTFE loading were studied. Toray 060, 090 and 120 samples which have different thicknesses of 220, 300 and 380 $\mu \mathrm{m}$, respectively, were examined to investigate the effect of this quantity as well. The Toray GDM materials were supplied by E-Tek with a PTFE coating applied. SGL10 GDMs were obtained directly from SGL Carbon, also with a PTFE coating applied. The physical properties of each GDM are listed in Table 1. The uncompressed thickness of each sample was measured directly prior to testing using a micrometer. Porosity values 
were taken from manufacturer specification sheets when available and from MIP data otherwise. The sample size used in the water-air capillary pressure testing and MIP testing were $20 \mathrm{~mm}$ diameter and $10 \mathrm{~mm} \times 30 \mathrm{~mm}$, respectively. Although this is certainly large enough to obtain a representative sample of material in terms of pore size distribution or porosity, it is small compared to the sheet they were taken from $(20 \mathrm{~cm} \times$ $20 \mathrm{~cm}$ for Toray materials and $21 \mathrm{~cm} \times 30 \mathrm{~cm}$ for SGL materials). Consequently, any heterogeneity in PTFE application or fiber distribution on the scale of the sheet would not be represented in the tested samples, meaning that the samples may not have been representative of any larger scale features. Nonetheless, numerous tests were performed on different samples from each sheet and very consistent results were observed between samples, although a controlled study of sample variability was not performed. 


\section{Results and Discussion}

Due to the effects of interfacial energy, mechanical equilibrium of any curved surface separating two fluid phases can be attained if and only if a difference exists between the fluid pressure acting locally on either side of the separating surface. This pressure difference (the capillary pressure $P_{C}$ ) is related to the surface tension $\sigma$ and mean curvature $H$ of the separating surface (interface) through the Young-Laplace equation $[24,40]:$

$$
P_{C}=2 \sigma H=\sigma \nabla \cdot \vec{n}
$$

where $\vec{n}$ is the unit normal to the surface. Capillary equilibrium of water and air within the pores of a GDM can be established over a broad range of water saturations $S_{W}$ by changing the phase pressure difference $P_{L}-P_{G}$ in a stepwise fashion. In the absence of hydrostatic gradients, the mean curvature $H$ of static water-air interfaces is spatially uniform and $P_{L}-P_{G}=P_{C}$. Defined in this manner, $P_{C}>0$ is conventionally understood to mean that water is the non-wetting phase [24]. It should be born in mind that Eq. (4) is a differential equation valid locally at each point on the interface. The shape of capillary surfaces in contact with a solid phase (and therefore $H$ ) is a solution to Eq. (4), subject to a boundary condition at the three-phase contact given by the contact angle. Importantly, the resulting functional relationship $P_{C}\left(S_{W}\right)$ between capillary pressure and water saturation in porous media is not unique [24]. As with other porous materials, permanent capillary pressure hysteresis in water-air-GDM systems $[18,20,21]$ is observed because capillary equilibrium is reached via a sequence of non-equilibrium interfacial configurations which differ depending on the history of saturation change [24]. The observed hysteresis in GDM materials may not be attributed entirely to contact angle 
hysteresis, as some have suggested [41]. Local pore geometry plays a decisive role since the shape of water-air interfaces at equilibrium (thus the magnitude and sign of $H$ ) is the solution to Eq. (4) subject to a boundary condition provided by the contact angle. Anticlastic interfaces for which $H<0$, even when $\theta>90^{\circ}$, can explain the condition whereby $P_{C}<0$ during water withdrawal [20].

\subsection{Comparison to other methods}

Several methods for measuring water-air capillary properties of GDMs have been recently presented. In some cases it is possible to compare the results of the present method with other reported methods where similar materials were tested. Figure 3a compares the present data to data obtained using MSP with water [9] and the data of Gallagher et al. [23] on untreated Toray (090 or 060 with $0 \mathrm{wt} \%$ PTFE). MSP is only able to scan in the direction of decreasing water saturation and so gives a limited picture of the full capillary pressure behavior. The present data and that from MSP are in poor agreement, with MSP showing water withdrawal requiring significantly more negative capillary pressures. MSP also shows residual water approaching zero at very negative capillary pressures (not shown in Figure 3a for clarity), compared with $6 \%$ in the present experiment. These discrepancies can be attributed to the fact that evaporation of water is not equivalent to forced displacement of water by air, a fundamental assumption of MSP, unless water is highly wetting and able to redistribute throughout the media [42]. This is unlikely in GDMs because of the weak affinity of water for either graphite or PTFE and consequently it can be expected that liquid water becomes highly disconnected during an MSP test. As pointed out previously [9] there is no way to differentiate between connected and disconnected water in MSP, since any water that becomes disconnected is 
still able to evaporate and leave the sample. This not only explains why MSP shows zero residual water saturation, but also explains the discrepancies in Figure 3a since the amount of water in the sample is always higher than the amount in equilibrium with the standard. The present data are in much better agreement with the water withdrawal curves of Gallagher et al. [23], who used a method based on water displacement. Gallagher et al. [23] show a non-zero residual saturation in close agreement with the value found here. Also noteworthy is that $S_{W}<1$ at $P_{C}=0$ in both the MSP and Gallagher et al. [23] experiments. This fact has been mistakenly interpreted ([9], [14]) as representing hydrophobic pore volume from which water is spontaneously ejected. Particularly because the data in Figure 3a are for GDMs with no PTFE loading, it seems more probable that this is an artifact resulting from the fact that in both the MSP and the Gallagher et al. [23] methods saturation is determined by direct weighing of the GDMs, which requires handling and removal of excess water. The method employed in the present experiments does not disturb the sample and spontaneous ejection of water is never observed (see also [20]). Figure $3 \mathrm{~b}$ compares results from the present method and the one of Harkness et al. [21] for Toray 060 with PTFE treatment. The agreement is excellent on the primary injection curves, with both the low pressure feature and the main leg of water injection matching very closely. Withdrawal curves do not agree as closely, but they are qualitatively similar in shape and both start very abruptly. Harkness et al. [21] show no residual saturation on water withdrawal, volume-controlled methods are not suitable for scanning saturation plateaus [38] and this observation is likely an artifact. Overall, the present method agrees well with the results of Harkness et al. [21] and Gallagher et al. [23]. Further discussion of these and other methods and comparisons 
between them may be found in Gostick et al. [38].

\subsection{Effect of Hydrophobic Coating}

\subsubsection{Mercury Intrusion Tests}

The application of a hydrophobic polymer coating to the internal surfaces of the GDM is intended to affect the resulting water-air capillary pressure curve by altering the surface wettability. Although the addition of PTFE to GDM substrates results in a small reduction of the GDM porosity (see Table 1), the results of MIP tests shown in Figure 4 for the Toray 090 (left) and SGL10 series (right) GDMs reveal no significant effects of PTFE loading on pore size distribution. Small differences observed in the MIP data for Toray 090 are not correlated to the amount of hydrophobic coating applied and so can be attributed to variability between samples.

\subsubsection{Water-Air Capillary Tests}

Figure 5 shows the water-air capillary pressure curves for Toray materials with different PTFE loadings and different uncompressed thicknesses. The untreated samples (Figure 5a-c) all show a large shoulder in the low pressure region $(<5000 \mathrm{~Pa})$ of the primary injection curves. The thinner GDM (i.e. Toray 060A) exhibits much higher saturation than the thicker materials at a given capillary pressure. This is the result of finite size effects since a significant amount of pore volume can be accessed from the surface prior to water breakthrough or percolation $[43,44]$. Contrary to very large (quasi-infinite) media where the vast majority of pores are internal, GDMs are so thin that invasion of surface pores, which unlike internal pores are not subject to accessibility limitations, represents a significant fraction of pore volume. The filling of pore volume at low 
pressure (i.e. below the percolation threshold) impacts the shape of the capillary pressure curve. Clearly, finite size effects will play a more prominent role in thinner materials since the proportion of pore volume accessible from the surface is greater. To further explore finite size effects in GDMs, the capillary curves for stacks of three GDMs were also obtained. This tripled the pore volume of the sample while maintaining the area of the injection face constant. As evident in Figure 6, measurements of the three-layer samples show that the capillary pressure curves become independent of the thickness of the individual layers comprising the three-layer stack for both treated and untreated GDMs. The persistence of a small feature in the very low pressure region $(\leq 2000 \mathrm{~Pa})$ is likely due to gaps between the sample and the hydrophilic membrane caused by macroscopic roughness and undulations on the surface of the GDM. The results of the three-layer stacks agree closely with the results for a single layer of Toray120, the thickest sample, indicating that finite size effects become negligible for $\delta \geq 400 \mu \mathrm{m}$. The role of finite-size effects on fuel cell performance is uncertain. Clearly, a thinner GDM will offer less gas phase transport resistance by virtue of a shorter diffusion length between the gas channel and catalyst layer. A thinner GDM, however, when subjected to invasion of liquid water will have a higher average saturation due to an increased fraction of pore volume occupied by dead-end (non-percolating) clusters of water-invaded pores. Dead-end clusters will exist in GDMs of all thickness, but in thinner materials they will extend proportionally farther into the layer and this may have consequences for lateral gas phase transport and the effectiveness of gas distribution under the lands.

Figure 7 plots all the data from Figure 5 together for direct comparison, with the 
secondary injection curves omitted for clarity. As can be seen, the primary water injection curves for all the untreated Toray samples converge at higher pressures $(>5000$ $\mathrm{Pa}$ ), indicating that the pore size distribution for each sample is similar despite the difference in thickness. The secondary injection curves for the untreated samples all show some spontaneous water imbibition (Figure 5), as was also observed by Harkness et al. [21] and Fairweather et al. [18]. The tendency of untreated samples to imbibe significant amounts of water could possibly explain why untreated GDMs perform poorly in fuel cells. The curves in Figure 5 also show that secondary injection occurs at somewhat lower pressures than the primary injection. This was also observed by Harkness et al. [21], but a definitive explanation for this effect is lacking. The amount of residual water, defined as the water trapped in the sample with no connection to the water reservoir while traversing a path of decreasing water saturation, seems insufficient to account for the increased ease with which water can reenter the sample. The possibility that exposure to water affects the surface of the fibers has not been investigated. As noted elsewhere [20], all subsequent water injections follow the secondary injection path exactly and this path is likely more relevant to operating fuel cells than primary injection data. It is of interest to determine if primary injection curves can be recovered (i.e. by drying) or if the alteration is permanent, since this has relevance to GDM degradation. Further work in this direction is needed.

The PTFE-treated Toray samples shown in Figure 5d-f also show a shoulder in the low pressure region of the primary injection curve that can be attributed to finite size effects, although it is less prominent than in the case of the untreated samples. Since the MIP 
results indicate that the pore size distributions are not significantly altered by PTFE, the differences must be due to changes in wettability brought about by the presence of PTFE. The effect of PTFE addition in these samples is clearly evident when compared directly to the untreated samples in Figure 7. The water injection curves for the treated samples converge onto a single curve at higher capillary pressures (> $7000 \mathrm{~Pa})$; significantly higher pressure than required for the untreated samples $(\approx 5000 \mathrm{~Pa})$. None of the PTFEtreated samples exhibit any spontaneous imbibition whatsoever on the secondary injection curves. This characteristic possibly plays a role in determining whether a GDM will perform well in a fuel cell or not. It should be pointed out that one of the treated Toray samples (120C) contains only 10\% PTFE loading, while the others (090D and 060D) contain 20\%. Nonetheless, the data obtained for the $120 \mathrm{C}$ material coincide with the data for 060D and 090D. This would indicate that doubling the PTFE loading does not significantly increase the hydrophobicity, suggesting that the additional PTFE makes thicker surface coatings rather than creating more hydrophobic surface area as intended.

Figure 5 also shows the response during the water withdrawal portion of the tests. Water is withdrawn from the PTFE coated samples at much less negative capillary pressures than from the untreated ones, indicating that the PTFE addition does indeed render the GDMs more hydrophobic. This is clearly demonstrated by the direct comparisons in Figure 7. Also observable in the water withdrawal curves is the presence of some residual liquid water at very negative capillary pressure for all samples. Sample thickness appears to have no significant effect on the water withdrawal curves, unlike the situation during water injection. The absence of an effect of thickness indicates that a 
different kind of capillary instability is operative during water withdrawal than during water injection [45]. During water injection, air is displaced from increasingly smaller pores as the capillary pressure is increased. Capillary pressures for water injection are controlled by the geometry of local constrictions (pore throats) within the GDM pore space. At the maximum pressure reached ( $c a .30,000 \mathrm{~Pa}$ ), only a very small volume of residual air remains, which is confined to the roughness features of the solid surfaces. This residual air maintains connectivity throughout the sample, however, and is always able to escape even as the water saturation approaches unity. If this were not the case, as Sinha et al. [5] have assumed, then clusters of air-filled pores would be trapped during water injection and it would not be possible for water to completely fill the GDM as observed experimentally in all cases. The fact that the gas phase maintains its connectivity is important for understanding the pore-level mechanisms of water withdrawal. Unlike water injection, where water can only enter pores that are accessible from the injection face, air has access to any pore in the sample during water withdrawal. The experimental observations are not consistent with the view that water withdrawal proceeds as an invasion percolation process, which would be subject to surface effects. Rather, observations are consistent with the view that water is withdrawn via a sequence of capillary instabilities that have been previously described as co-operative pore filling [45] - a mechanism prevalent in porous materials of low pore body size-to-pore throat size aspect ratio like GDMs. The experimental results reported here cast doubt on the earlier suggestion that water withdrawal is equivalent to drainage of a wetting phase [9], a suggestion which is also inconsistent with experimental observations of trapped water saturation after water withdrawal. 
Figure 8 shows the primary injection and withdrawal curves for the SGL10 series GDMs containing varying amounts of PTFE. The effect of PTFE addition on these curves does not follow as consistent a trend as in the case of the Toray materials. The water injection curves of the treated samples do not correlate with PTFE content. Water withdrawal curves behave in a more predictable manner with the sample containing the largest amount of PTFE (10CA) appearing to be the most hydrophobic, but the differences between treated and untreated samples are not as strong as for the Toray materials. Clearly, the responses of the 10BA and 10CA samples exhibit some differences that cannot be explained by the amount of PTFE alone. It is possible that these are due to some variations in the way PTFE is distributed throughout the GDMs.

\subsection{Effect of Sample Compression}

GDM compression is an inevitable feature of fuel cell assembly. Stacks must be compressed to ensure that a tight gas seal is formed and that good electrical contact between the bipolar plates, GDMs and catalyst layers is made. However, compression of the GDM reduces its porosity and pore sizes. The effect of compression on permeability $[39,46]$ and on effective diffusivity $[47,48]$ has been investigated and shown to be very significant. Since capillary pressure curves are also affected by reduction in pore sizes, this effect has begun to receive attention. Harkness et al. [21] measured capillary pressure curves with various compressive loads applied, but only for a single sample. Kumbur et al. [49] studied compressed samples using the MSP approach, but this method is limited to water withdrawal only. 
The method used in the present study was adapted to compress samples during measurements. Figure 9 shows the capillary pressure curves for SGL10AA (top) and SGL10BA (bottom) with and without compression. These data are scaled with respect to the compressed thickness so the saturation approaches unity at high capillary pressures for both samples. Dramatic differences due to the influence of compression can be seen in the water entry and withdrawal pressures for both treated and untreated samples. When the sample is compressed, the injection pressure is increased as expected due to the smaller resulting pore sizes. Also noteworthy is the absence of a shoulder in the low pressure region for the compressed samples. It is possible that compression preferentially reduces pore size at the surfaces of the GDM by compacting fibers more tightly, but it is also likely that compression smoothens out the undulations and macroscopic roughness of the surface, closing any gaps that may have existed between the sample and the hydrophilic membrane. Figure 9 also shows that water withdrawal takes place at more negative values of the capillary pressure when the samples are compressed, meaning that more work needs to be expended in order to force water out of the GDM.

Figure 10 shows the water-air capillary pressure curves for untreated (top) and treated (bottom) Toray 120 samples with and without compression. These curves show the same general trends as for the SGL10 materials in Figure 9. Water injection into the compressed samples occurs at higher capillary pressures, indicating that pore sizes have become smaller. As with the SGL materials, the low pressure shoulder is largely eliminated as well. Unlike what was observed with the SGL10 samples, water 
withdrawal pressures for the Toray 120 samples are observed to change only slightly under compression. This may be due to the known significant differences in fiber arrangement and conformation between the two kinds of GDM.

\subsection{Assessment of GDM Wettability}

The addition of hydrophobic polymer coatings to GDMs clearly alters their wettability, but a convenient quantitative indicator of the effect of this treatment has not heretofore been used. One approach to quantifying this effect is to use a wettability index obtained empirically from capillary curves [25]. Of several wettability index definitions proposed, the USBM index $\left(I_{U S B M}\right)$ is most appropriate because it is very sensitive near neutral wettability [25]. The $I_{U S B M}$ is based on the observation that the area under a capillary pressure curve corresponds to the amount of work required to inject or withdraw a fluid from a porous medium.[34,50] Injection of a non-wetting fluid will require more work than its removal, since the latter is not opposed by capillary forces. Calculation of the $I_{U S B M}$ requires determining the areas $A_{1}$ and $A_{2}$ as shown in Figure 11 and inserting them into Eq. (5) as follows:

$$
I_{U S B M}=\log \left(\frac{A_{1}}{A_{2}}\right)
$$

Defined in this way, negative $I_{U S B M}$ values signify that the material tends to be hydrophobic, while positive values indicate a hydrophilic tendency. The magnitude of $I_{U S B M}$ indicates the extent of wettability. A value near zero corresponds to intermediate (neutral) wettability, while more positive or negative values signify increasing or 
decreasing wetting preference for water, respectively [25].

Values of $I_{U S B M}$ have been determined for all of the GDM materials tested, with and without compression, and are given in Figure 12. This analysis reveals a notable difference in wettability when PTFE is added, something that could not be easily discerned from visual inspection of the capillary pressure curves. Before a PTFE layer is applied, all untreated samples have a positive $I_{U S B M}$; whereas subsequent application of PTFE causes $I_{U S B M}$ for all samples to become negative.

It might appear surprising that the wettability index values change when a sample is compressed (see Figure 11). Since compression does not alter the chemical constitution of the GDM, the contact angle should remain unchanged. The fact that significant changes are observed illustrates the fact that capillary behavior is controlled by structural features as much as by surface wettability (measured by contact angle). The significance of the structure in determining the magnitude and sign of the mean curvature of fluid interfaces (viz. Eq. (4)) is greater in porous media of intermediate surface wettability. As $\theta \rightarrow 90^{\circ}$, the validity of capillary tube models to describe wettability effects on capillary pressure [41] is challenged [26]. Such models are based on Washburn's equation, which is valid for straight cylindrical capillaries of radius $R$ [24]:

$$
P_{C}=\frac{2 \sigma \cos (\theta)}{R}
$$

According to Eq. (6), the sign of the capillary pressure is decided by the value of $\cos (\theta)$. Were this model valid, $\theta=90^{\circ}$ would define a transition between water being the wetting 
or non-wetting phase within a porous medium, and the concept of hydrophilichydrophobic duality for PTFE-treated GDM would be justified on grounds that $\theta \approx 75^{\circ}-$ $86^{\circ}$ for water on graphite $[8,27,28]$ and $\theta \approx 108^{\circ}-112^{\circ}$ for water on PTFE $[29,30]$. In real porous media, interconnectivity and the complexity of pore geometry render $\cos (\theta)$ a poor predictor of the effect of wettability on capillary pressure when $\theta \rightarrow 90^{\circ}$ [25]. Eq. (6) erroneously predicts spontaneous water uptake when $\theta<90^{\circ}$, when in fact spontaneous imbibition of liquids in porous media has been shown to require contact angles less than 40 to $50^{\circ}[51,52]$. When describing effects of wettability on capillary pressure of porous media, it is necessary to distinguish between systems of strong (hydrophilic/hydrophobic) and neutral (intermediate) wettability. According to Anderson [25] a system is hydrophilic (water-wet) when $\theta$ is between 0 and 60 to $75^{\circ}$ and hydrophobic when $\theta$ is between 180 and 105 to $120^{\circ}$. When contact angles lie in the intermediate range, as in the case of native and PTFE-treated GDMs, it is more appropriate to classify the system as being neutrally or intermediately wet. Since Eq. (6) fails to describe the effects of wettability on capillary pressure when $\theta \rightarrow 90^{\circ}$, the notion that hydrophilic and hydrophobic pore networks are present within PTFE-treated GDM is of little practical value.

For the Toray samples, compression shifts the wettability index to more negative values. Such a shift means that water injection requires more work than withdrawal compared to the uncompressed materials. This is explained by the fact that water injection is a drainage process and is controlled strictly by pore throat sizes, while water withdrawal is controlled by capillary instabilities in which pore body sizes and cooperative pore filling 
play an important role [45]. The shift in wettability index suggests that pore throat size is reduced more noticeably than the pore body size for Toray 120. The SGL materials, on the other hand, behave differently upon compression. The shift of the index for the untreated sample (SGL10AA) corresponds to the material becoming apparently more hydrophilic, while the index for the treated sample (SGL10BA) remains virtually unchanged. It is not clear why the SGL materials behave differently, but it is possible that the curved and entangled fibers of the SGL10 GDMs respond in a much more complex fashion upon compression. The compression-induced change in apparent wettability may have implications for non-uniform water accumulation in a working fuel cell, since compression of the GDM during assembly does not occur uniformly, but is restricted to the regions under the channel ribs or lands [53]. 


\section{Conclusions}

A recently reported water-air capillary pressure measurement technique [20] was exploited to study the capillary properties of a wide range of GDM materials with different thicknesses, PTFE loadings and compressions. All samples showed a significant hysteresis whereby water injection occurred at positive capillary pressure while water withdrawal occurred at negative capillary pressure, in agreement with recent reports $[18,21]$. The data from very thin samples $(\sim 200 \mu \mathrm{m})$ showed a large shoulder in the low pressure range due to finite size effects. This feature was diminished in the curves for thicker samples. Comparison of identical substrate materials with and without a hydrophobic polymer coating showed significant differences. In materials with hydrophobic treatment, much higher pressure was required for water injection, whereas water withdrawal occurred at considerably less negative capillary pressure. No difference was observed between the curves for samples containing 10 and $20 \mathrm{wt} \%$ PTFE, suggesting that there was no advantage to adding more polymer. MIP tests conducted on samples with varying amount of PTFE loading showed virtually no difference, confirming that differences in the water-air capillary pressure curves were due to wettability changes rather than structural changes caused by the addition of PTFE. Measurements conducted to investigate the influence of compression revealed significant changes in the capillary behavior. Water injection into Toray materials was shifted to significantly higher pressure, while the withdrawal pressures remained relatively unchanged. SGL materials showed strong shifts toward more positive pressure for water injection and more negative pressure for water withdrawal. Finally, the observed capillary pressure data were analyzed in terms of the USBM wettability index to provide 
a quantitative description of the extent of GDM wettability. It was found that all untreated samples exhibited a positive $I_{U S B M}$ value, but then shifted to negative values after being treated with PTFE. The IUSBM values of the GDMs changed upon compression, highlighting the fact that GDM capillary behavior is highly influenced by pore structure. In all cases, the tested GDMs could be characterized as materials of intermediate (neutral), rather than mixed, wettability with regards to the water-air fluid pair

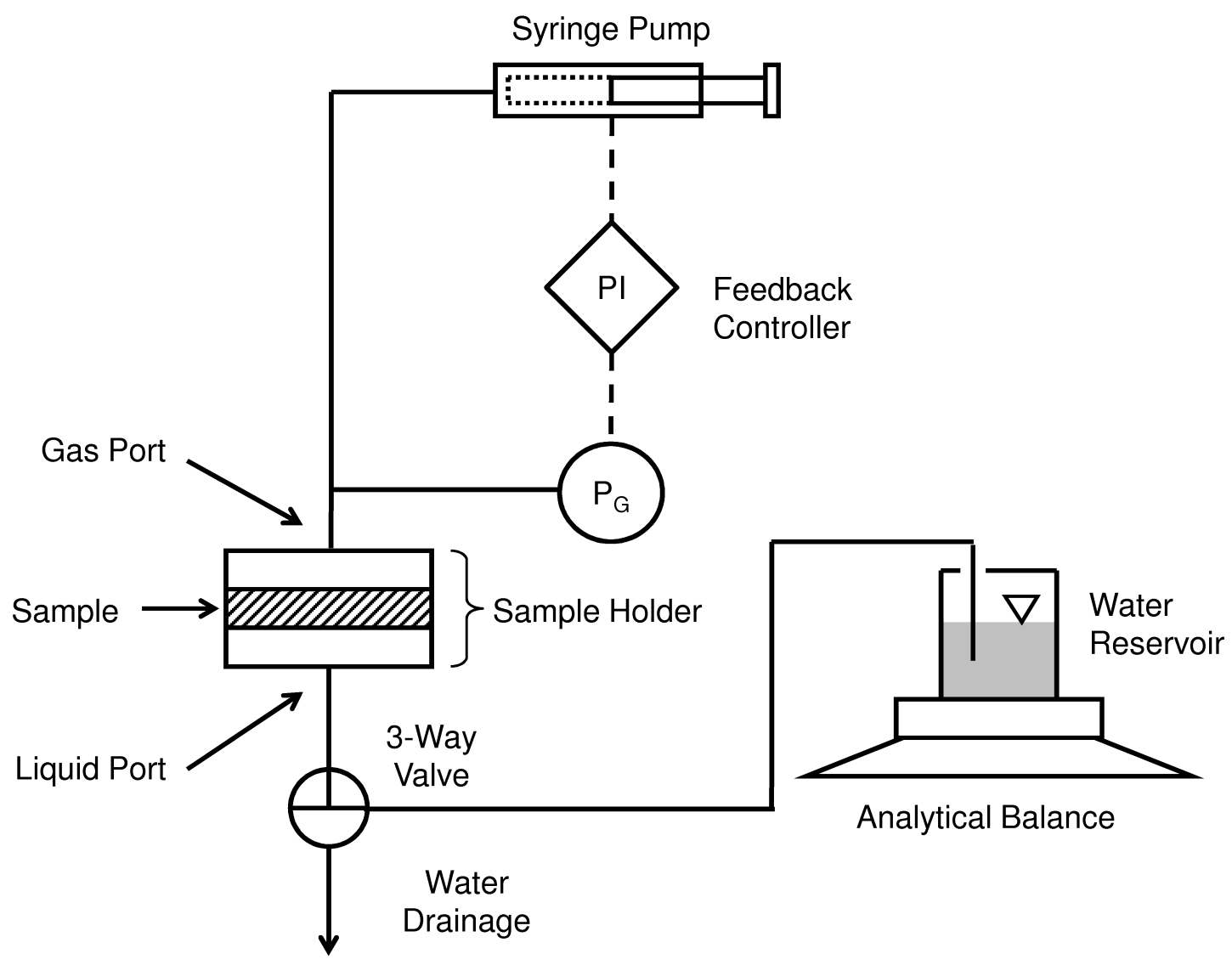




\section{Acknowledgments}

The authors wish to thank the Natural Science and Engineering Research Council of Canada for financial support throughout the course of this project. 


\section{Appendix A. Capillary Pressure Curve Correlations}

Analytic functions for the empirical representation of all water-air capillary pressure curves obtained in this work are provided in this Appendix. To describe the capillary pressure data, a van Genuchten-type equation [58] is adopted with some modifications to accommodate features specific to GDMs, such as the apparent switch in wettability and finite size effects. Three different fitting functions are provided for each material to represent each of primary injection, primary withdrawal and secondary injection capillary pressure data. The primary and secondary injection curves display two inflection points, whereas the withdrawal curves only show a single inflection point. To accommodate both of these behaviors an expression was devised that blends $n \geq 1$ individual curves, allowing a curve with any number of inflection points to be described:

$$
S_{W}=\sum_{i=1}^{n} f_{(i)}\left[S_{W(i)}\left(S_{W, M}-S_{W, R}\right)+S_{W, R}\right]
$$

where $f_{(i)}$ is the proportion of the $i^{t h}$ curve contributing to the total curve, $S_{W, R}$ is the residual water saturation at highly negative capillary pressure and $S_{W, M}$ is the maximum water saturation achieved at large positive capillary pressure. Values of $f_{(i)}, S_{W, R}$ and $S_{W, M}$ are tabulated in Table 2 for each material. $S_{W(i)}$ is the saturation given by the van Genuchten equation using the $i^{\text {th }}$ fitting parameters. For the primary and secondary injection it is given by:

$$
S_{W(i)}=1-\left(1+\left(\frac{P_{C}+P_{A T M}^{S T D}}{P_{C, b(i)}}\right)^{n_{(i)}}\right)^{-m_{(i)}}
$$

where the fitting parameters $P_{C, b(i)}, m_{(i)}$, and $n_{(i)}$ are listed in Table 2. Since the capillary pressure spans both positive and negative values, it was necessary to offset $P_{C}$ by adding 
$P_{A T M}^{S T D}$ to the capillary pressure. Primary water withdrawal is modeled using Eq. (7) with $n=1$ since these curves show only a single inflection point. In order to plot the withdrawal curves on the same axes as the injection curves a modification to Eq. (8) is necessary, as follows:

$$
S_{W(i)}=\left(1+\left(\frac{-P_{C}+P_{A T M}^{S T D}}{P_{C, b(i)}}\right)^{n_{(i)}}\right)^{-m_{(i)}}
$$

Eq. (9) is equivalent to Eq. (8) mirrored about both the $S_{W}$ and $P_{C}$ axes. As can be seen in Figure 9 and Figure 10, the quality of the fits is fairly good for most of the range, with some deviation in the high saturation range $\left(S_{W}>0.9\right)$. 


\section{References}

1. J.O.M. Bockris, Science, 176 (1972) 1323.

2. M.Z. Jacobson, W.G. Colella, D.M. Golden, Science, 308 (2005) 1901-1905.

3. M.F. Mathias, R. Makharia, H.A. Gasteiger, J.J. Conley, T.J. Fuller, C.J. Gittleman, S.S. Kocha, D.P. Miller, C.K. Mittelsteadt, T. Xie, S.G. Van, P.T. Yu, Electrochem. Soc. Interface, 14 (2005) 24-35.

4. $\quad$ M.S. Wilson, S. Goffesfeld, J. Electrochem. Soc., 139 (1992) 28-30.

5. P.K. Sinha, P.P. Mukherjee, C.Y. Wang, J. Mater. Chem., 17 (2007) 3089-3103.

6. C. Lim, C.Y. Wang, Electrochim. Acta, 49 (2004) 4149-4156.

7. M.F. Mathias, J. Roth, J. Fleming, W. Lehnert, Diffusion media materials and characterization, in Handbook of Fuel Cells - Fundamentals, Technology and Applications. Volume 3, Part 1., W. Vielstich, H.A. Gasteiger A. Lamm, Editors. 2003, John Wily \& Sons: New York. p. 517-537.

8. A.W. Adamson, Physical Chemistry of Surfaces. 1982, New York: John Wiley \& Sons.

9. J.T. Gostick, M.W. Fowler, M.A. Ioannidis, M.D. Pritzker, Y.M. Volfkovich, A. Sakars, J. Power Sources, 156 (2006) 375-387.

10. V. Gurau, M.J. Bluemle, E.S. De Castro, Y.M. Tsou, J.A. Mann, T.A. Zawodzinski, J. Power Sources, 160 (2006) 1156-1162.

11. M.V. Williams, E. Begg, L. Bonville, H.R. Kunz, J.M. Fenton, J. Electrochem. Soc., 151 (2004) 1173-1180.

12. H. Dohle, H. Schmitz, T. Bewer, J. Mergel, D. Stolten, J. Power Sources, 106 (2002) 313-322.

13. J. Benziger, J. Nehlsen, D. Blackwell, T. Brennan, J. Itescu, J. Membrane Sci., 261 (2005) 98-106.

14. E.C. Kumbur, K.V. Sharp, M.M. Mench, J. Electrochem. Soc., 154 (2007) B1295B1304.

15. Y.M. Volfkovich, V.E. Sosenkin, N.F. Nikol'skaya, T.L. Kulova, Russ. J. Electrochem., 44 (2008) 278-285.

16. M.J. Martinez, S. Shimpalee, J.W. Van Zee, A.V. Sakars, J. Electrochem. Soc., 156 (2009) B558-B564.

17. T. Koido, T. Furusawa, K. Moriyama, J. Power Sources, 175 (2008) 127-136.

18. J.D. Fairweather, P. Cheung, J. St Pierre, D.T. Schwartz, Electrochem. Commun., 9 (2007) 2340-2345.

19. T.V. Nguyen, G. Lin, H. Ohn, X. Wang, Electrochem. Solid-State Lett., 11 (2008) B127-B131.

20. J.T. Gostick, M.A. Ioannidis, M.W. Fowler, M.D. Pritzker, Electrochem. Commun., 10 (2008) 1520-1523.

21. I.R. Harkness, N. Hussain, L. Smith, J.D.B. Sharman, J. Power Sources, In Press.

22. D. Rensink, S. Fell, J. Roth. Liquid water transport and distribution in fibrous porous media and gas channels. in Proceedings of the Sixth International Conference on Nanochannels, Microchannels and Minichannels. 2008. Darmstadt, Germany.

23. K.G. Gallagher, R.M. Darling, T.W. Patterson, M.L. Perry, J. Electrochem. Soc., 
155 (2008) B1225-B1231.

24. F.A.L. Dullien, Porous Media: Fluid Transport and Pore Structure. 1992, New York: Academic Press.

25. W.G. Anderson, J. Petro. Tech., 38 (1986) 1246-1262.

26. W.G. Anderson, J. Petro. Tech., 39 (1987) 1283-1300.

27. A.V. Neimark, J. Adhes. Sci. Technol., 13 (1999) 1137-1154.

28. D. Mattia, H.H. Ban, Y. Gogotsi, Langmuir, 22 (2006) 1789-1794.

29. S. Goswami, S. Klaus, J. Benziger, Langmuir, 24 (2008) 8627-8633.

30. N.R. Morrow, J. Can. Pet. Technol., 15 (1976) 49-69.

31. A.Z. Weber, R.M. Darling, J. Newman, J. Electrochem. Soc., 151 (2004) A1715A1727.

32. R.P. Ramasamy, E.C. Kumbur, M.M. Mench, W. Liu, D. Moore, M. Murthy, Int. J. Hydrogen Energy, 33 (2008) 3351-3367.

33. S. Park, B.N. Popov, Electrochim. Acta, 54 (2009) 3473-3479.

34. N.R. Morrow, Ind. Eng. Chem., 62 (1970) 32.

35. P.G. Toledo, L.E. Scriven, H.T. Davis, SPE Formation Evaluation, 9 (1994) 46-54.

36. J.D. Smith, I. Chatzis, M.A. Ioannidis, J. Can. Pet. Technol., 44 (2005) 25-31.

37. M.A. Knackstedt, A.P. Sheppard, W.V. Pinczewski, Phys. Rev. E, 58 (1998) R6923-R6926.

38. J.T. Gostick, M.A. Ioannidis, M.W. Fowler, M.D. Pritzker, Characterization of the Capillary Properties of Gas Diffusion Media, in Modern Aspects of Electrochemistry, C.Y. Wang U. Pasaogullari, Editors. In Press, Springer: Berlin.

39. J.T. Gostick, M.W. Fowler, M.D. Pritzker, M.A. Ioannidis, L.M. Behra, J. Power Sources, 162 (2006) 228-238.

40. A. Defay, I. Prigogine, A. Bellemans, Surface Tension and Adsorption. 1966, New York: John Wiley \& Sons.

41. P. Cheung, J.D. Fairweather, D.T. Schwartz, J. Power Sources, 187 (2009) 487492.

42. I.N. Tsimpanogiannis, Y.C. Yortsos, S. Poulou, N. Kanellopoulos, A.K. Stubos, Phys. Rev. E, 59 (1999) 4353-4365.

43. J.T. Gostick, M.A. Ioannidis, M.W. Fowler, M.D. Pritzker, Electrochem. Commun., 11 (2009) 576-579.

44. R.G. Larson, N.R. Morrow, Powder Technol., 30 (1981) 123-138.

45. M.A. Ioannidis, I. Chatzis, A.C. Payatakes, J. Colloid Interface Sci., 143 (1991) 22-36.

46. V. Gurau, M.J. Bluemle, E.S. De Castro, Y.M. Tsou, T.A. Zawodzinski, J.A. Mann, J. Power Sources, 165 (2007) 793-802.

47. D.R. Baker, C. Wieser, K.C. Neyerlin, M.W. Murphy, ECS Transactions, 3 (2006) 989-999.

48. D. Kramer, S.A. Freunberger, R. Fluckiger, I.A. Schneider, A. Wokaun, F.N. Buchi, G.G. Scherer, J. Electroanal. Chem., 612 (2008) 63-77.

49. E.C. Kumbur, K.V. Sharp, M.M. Mench, J. Electrochem. Soc., 154 (2007) B1305B1314.

50. J.C. Melrose, Ind. Eng. Chem., 60 (1968) 53-70.

51. N.J. Shirtcliffe, G. McHale, M.I. Newton, F.B. Pyatt, S.H. Doerr, Appl. Phys. Lett., 89 (2006). 
52. J.C. Melrose, SPE J, 5 (1965) 259-271.

53. I. Nitta, O. Himanen, M. Mikkola, Fuel Cells, 8 (2008) 111-119.

54. Y. Wang, J. Power Sources, 185 (2008) 261-271.

55. Y. Wang, C.Y. Wang, K.S. Chen, Electrochim. Acta, 52 (2007) 3965-3975.

56. M. Acosta, C. Merten, G. Eigenberger, H. Class, R. Helmig, B. Thoben, H. Muller-Steinhagen, J. Power Sources, 159 (2006) 1123-1141.

57. V. Gurau, T.A. Zawodzinski, J.A. Mann, J. Fuel Cell Sci. Tech., 5 (2008).

58. M.T. van Genuchten, Soil Sci. Soc. Am. J., 44 (1980) 892-898. 


\section{Nomenclature}

\begin{tabular}{|c|c|c|}
\hline Symbol & Description & Units \\
\hline$A$ & Area Under Capillary Pressure Curve (defined in Figure 11) & \\
\hline$d$ & Sample Diameter & $\mathrm{m}$ \\
\hline$\delta$ & Sample Thickness & $\mathrm{m}$ \\
\hline$\varepsilon$ & Porosity & $-/-$ \\
\hline$f$ & $\begin{array}{l}\text { Contribution of Individual van Genuchten Equation to Overall } \\
\text { Fitting Equation }\end{array}$ & $-/-$ \\
\hline$H$ & Interface Curvature & $m^{-1}$ \\
\hline$I_{U S B M}$ & US Bureau of Mines Wettability Index & $-/-$ \\
\hline$m$ & Constant in van Genuchten Equation & \\
\hline$m_{W}$ & Mass of Water Uptake into GDM & $\mathrm{kg}$ \\
\hline$n$ & Constant in van Genuchten Equation & \\
\hline$P_{C, b}$ & Constant in van Genuchten Equation & \\
\hline$P_{C}$ & Capillary Pressure, defined here as $P_{C} \equiv P_{L}-P_{G}$ & $\mathrm{~Pa}$ \\
\hline$P_{G}$ & Gas Pressure & $\mathrm{Pa}$ \\
\hline$P_{L}$ & Liquid Pressure & $\mathrm{Pa}$ \\
\hline$\theta$ & Contact Angle & $\circ$ \\
\hline$R$ & Pore Radius & $\mathrm{m}$ \\
\hline$\rho$ & Water Density & $\mathrm{kg} \cdot \mathrm{m}^{-3}$ \\
\hline$S_{W}$ & Water Saturation in GDM & \\
\hline
\end{tabular}


$\sigma$

$V_{p}$

$V_{W}$

Subscripts

C

0

M

$\mathrm{R}$

Pore Volume in GDM

$m^{3}$

Water Volume in GDM

$m^{3}$

Compressed

Uncompressed

Maximum Saturation at Large Positive Capillary Pressure

Residual Saturation at Large Negative Capillary Pressure 


\section{Figure and Table Captions}

Table 1: $\quad$ Physical properties of materials tested in this study

\begin{tabular}{ccccc}
\hline Brand & Model & $\begin{array}{c}\text { Thickness } \\
{[\mu \mathrm{m}]}\end{array}$ & $\begin{array}{c}\text { PTFE Loading } \\
{[\mathbf{w t} \%]}\end{array}$ & $\begin{array}{c}\text { Porosity } \\
{\left[\mathbf{m}^{3} / \mathbf{m}^{3}\right]}\end{array}$ \\
\hline SGL & 10AA & 390 & 0 & 0.90 \\
& 10BA & 360 & 5 & 0.88 \\
& 10CA & 400 & 10 & 0.86 \\
\hline Toray & 10DA & 410 & 20 & 0.84 \\
\hline & 060A & 220 & 0 & 0.78 \\
& 060C & 220 & 10 & 0.75 \\
& 090A & 290 & 0 & 0.78 \\
& 090C & 290 & 10 & 0.75 \\
& 090D & 290 & 20 & 0.73 \\
& 120A & 380 & 0 & 0.78 \\
& 120D & 380 & 20 & 0.73 \\
\hline
\end{tabular}

Table 2: $\quad$ Parameters used for fitting experimental data

\begin{tabular}{cccccccccc}
\hline & $\boldsymbol{P}_{c, b(1)}$ & $\boldsymbol{m}_{(1)}$ & $\boldsymbol{n}_{(1)}$ & $\boldsymbol{f}_{(1)}$ & $\boldsymbol{P}_{c, b(2)}$ & $\boldsymbol{m}_{(2)}$ & $\boldsymbol{n}_{(2)}$ & $\boldsymbol{f}_{(2)}$ & $\boldsymbol{S}_{W, R}$ \\
\hline SGL10AA(Uncompressed) & & & & & & & & & \\
Primary Injection & 102000 & 200 & 1 & 0.25 & 105000 & 200 & 0.6 & 0.75 & 0 \\
Secondary Injection & 102000 & 200 & 1 & 0.25 & 104000 & 200 & 0.6 & 0.75 & 0.08 \\
Primary Withdrawal & 103600 & 500 & 0.2 & 1 & --- & --- & --- & --- & 0.08 \\
\hline SGL10AA(Compressed) & & & & & & & & & \\
Primary Injection & 106000 & 150 & 0.5 & 1 & --- & --- & --- & --- & 0 \\
Secondary Injection & 104200 & 90 & 0.7 & 1 & --- & --- & --- & --- & 0.08 \\
Primary Withdrawal & 106500 & 450 & 0.1 & 1 & --- & --- & --- & --- & 0.08 \\
\hline SGL10BA(Uncompressed) & & & & & & & & & \\
Primary Injection & 102000 & 200 & 0.5 & 0.3 & 105000 & 200 & 0.3 & 0.7 & 0 \\
Secondary Injection & 102000 & 200 & 0.4 & 0.3 & 105000 & 200 & 0.5 & 0.7 & 0.06 \\
Primary Withdrawal & 104500 & 150 & 2 & 1 & --- & --- & --- & --- & 0.06 \\
\hline PGL10BA(Compressed) & & & & & & & & & \\
Primary Injection & 107000 & 80 & 0.5 & 1 & --- & --- & --- & --- & 0 \\
Secondary Injection & 107000 & 100 & 0.6 & 1 & --- & --- & --- & --- & 0.08 \\
Primary Withdrawal & 104500 & 150 & 0.3 & 1 & --- & --- & --- & --- & 0.08 \\
\hline Primary Injection & 103000 & 250 & 1 & 0.2 & 106000 & 200 & 1 & 0.8 & 0 \\
Secondary Injection & 103000 & 250 & 1 & 0.25 & 105500 & 200 & 1 & 0.75 & 0.07 \\
Primary Withdrawal & 104000 & 250 & 1 & 1 & --- & --- & --- & --- & 0.07 \\
\hline PGimary Injection & 102000 & 125 & 0.5 & 0.3 & 107500 & 200 & 2 & 0.7 & 0 \\
Secondary Injection & 101500 & 125 & 0.9 & 0.4 & 106000 & 150 & 1.5 & 0.6 & 0.08
\end{tabular}




\begin{tabular}{|c|c|c|c|c|c|c|c|c|c|}
\hline Primary Withdrawal & 107500 & 400 & 0.3 & 1 & --- & --- & --- & --- & 0.08 \\
\hline \multicolumn{10}{|l|}{ Toray120A(Compressed) } \\
\hline Primary Injection & 110000 & 75 & 2 & 1 & --- & --- & --- & --- & 0 \\
\hline Secondary Injection & 109000 & 75 & 2 & 1 & --- & --- & --- & --- & 0.08 \\
\hline Primary Withdrawal & 108000 & 175 & 0.5 & 1 & --- & --- & --- & --- & 0.08 \\
\hline \multicolumn{10}{|l|}{ Toray120C(Uncompressed) } \\
\hline Primary Injection & 103500 & 200 & 0.6 & 0.15 & 108000 & 200 & 0.5 & 0.85 & 0 \\
\hline Secondary Injection & 103500 & 100 & 0.5 & 0.25 & 107000 & 400 & 0.2 & 0.75 & 0.08 \\
\hline Primary Withdrawal & 105000 & 200 & 0.6 & 1 & --- & --- & --- & -- & 0.08 \\
\hline \multicolumn{10}{|l|}{ Toray120C(Compressed) } \\
\hline Primary Injection & 110000 & 135 & 0.3 & 1 & --- & --- & --- & --- & 0 \\
\hline Secondary Injection & 110000 & 125 & 0.4 & 1 & --- & --- & --- & --- & 0.07 \\
\hline Primary Withdrawal & 105500 & 175 & 0.6 & 1 & --- & --- & --- & --- & 0.07 \\
\hline \multicolumn{10}{|l|}{ Toray090A(Uncompressed) } \\
\hline Primary Injection & 102000 & 200 & 0.5 & 0.3 & 107000 & 200 & 1 & 0.7 & 0 \\
\hline Secondary Injection & 102000 & 150 & 0.7 & 0.4 & 106000 & 200 & 0.7 & 0.6 & 0.04 \\
\hline Primary Withdrawal & 107000 & 200 & 0.7 & 1 & --- & --- & --- & --- & 0.04 \\
\hline \multicolumn{10}{|l|}{ Toray090D(Uncompressed) } \\
\hline Primary Injection & 102000 & 200 & 0.6 & 0.2 & 108000 & 150 & 0.5 & 0.8 & 0 \\
\hline Secondary Injection & 102000 & 200 & 0.9 & 0.2 & 107600 & 200 & 0.6 & 0.8 & 0.13 \\
\hline Primary Withdrawal & 105000 & 250 & 0.8 & 1 & --- & --- & --- & --- & 0.13 \\
\hline \multicolumn{10}{|l|}{ Toray060A(Uncompressed) } \\
\hline Primary Injection & 102000 & 200 & 0.4 & 0.5 & 106500 & 200 & 0.6 & 0.5 & 0 \\
\hline Secondary Injection & 100000 & 100 & 0.6 & 0.5 & 105000 & 150 & 0.5 & 0.5 & 0.04 \\
\hline Primary Withdrawal & 107000 & 200 & 0.5 & 1 & --- & --- & --- & --- & 0.04 \\
\hline \multicolumn{10}{|l|}{ Toray060D(Uncompressed) } \\
\hline Primary Injection & 101500 & 250 & 0.3 & 0.25 & 108000 & 150 & 0.5 & 0.75 & 0 \\
\hline Secondary Injection & 101500 & 250 & 0.4 & 0.25 & 107500 & 200 & 0.5 & 0.75 & 0.1 \\
\hline Primary Withdrawal & 105000 & 150 & 1 & 1 & --- & --- & --- & --- & 0.1 \\
\hline
\end{tabular}




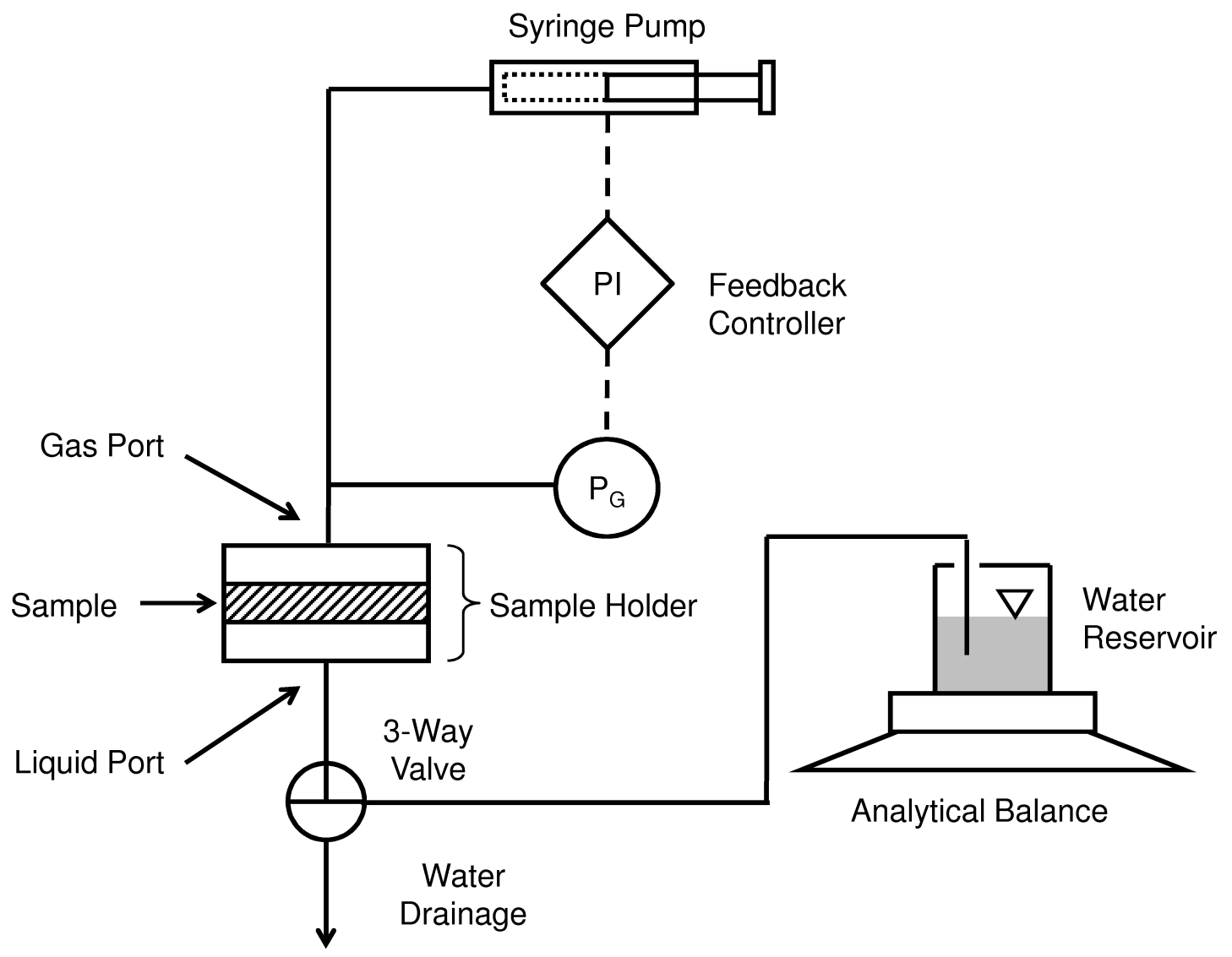

Figure 1: Schematic diagram of system setup. Sample holder is shown in more detail in Figure 2. 


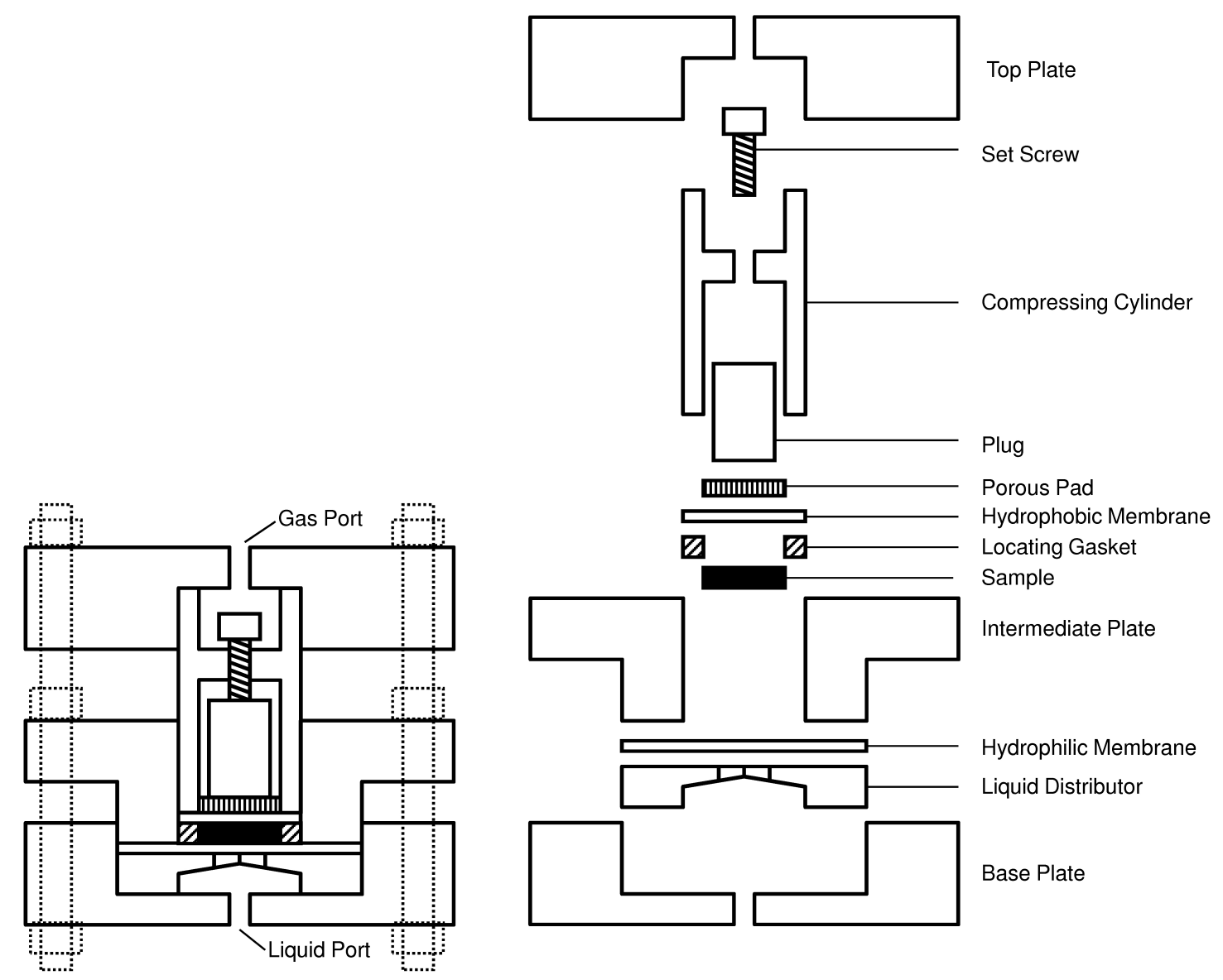

Figure 2: $\quad$ Sample holder. Left: assembled view. Right: Expanded view with parts labeled. The set screw is tightened after assembly by inserting a hex-key through the gas port. 


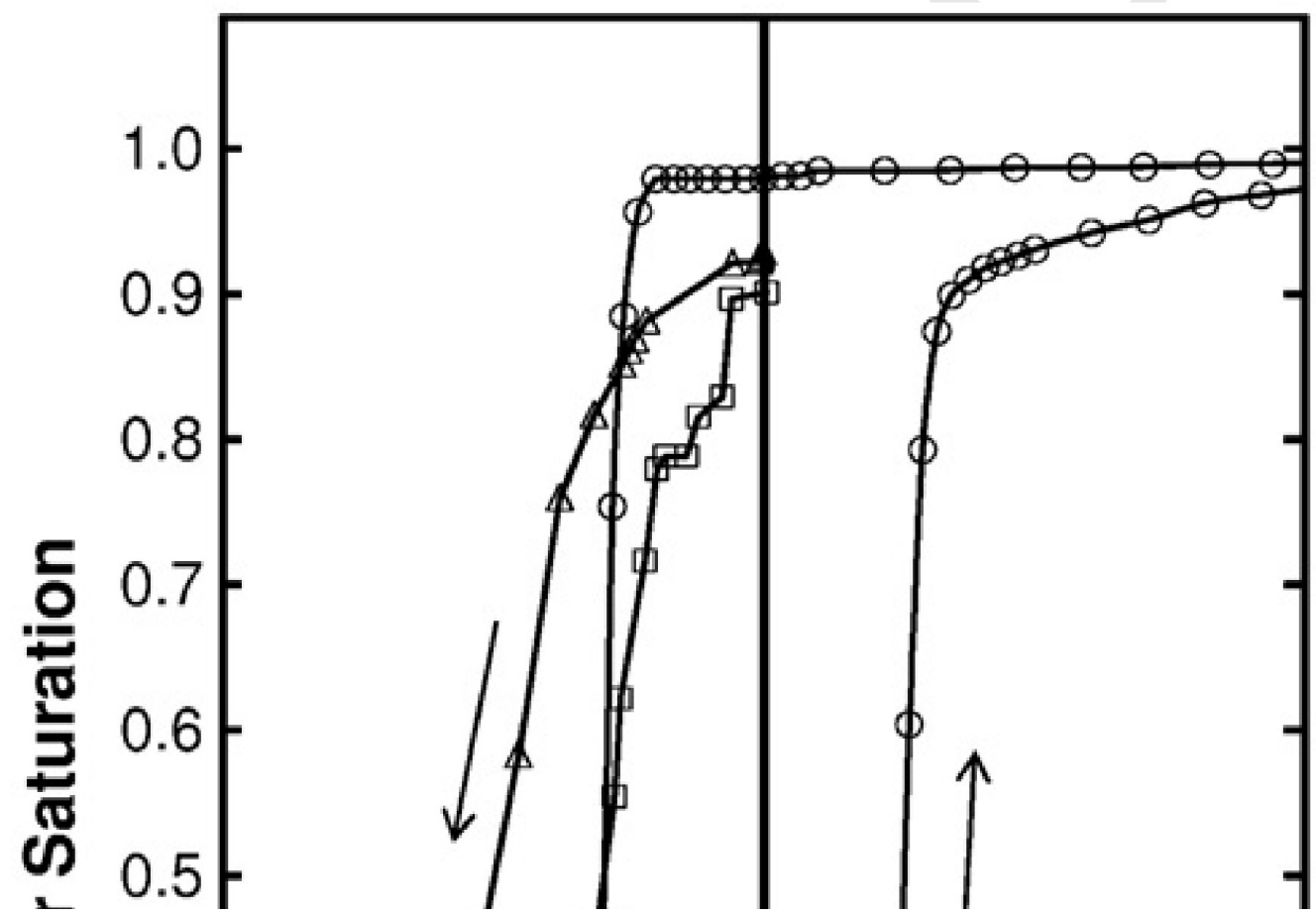

Figure 3: Comparison of the present method with reported literature data on similar materials. Left: Toray 090 with no PTFE treatment. Right: Toray 060 with PTFE treatment $(20 \mathrm{wt} \%$ in present experiments and $10 \mathrm{wt} \%$ in the experiments of Harkness et al. [21]). 

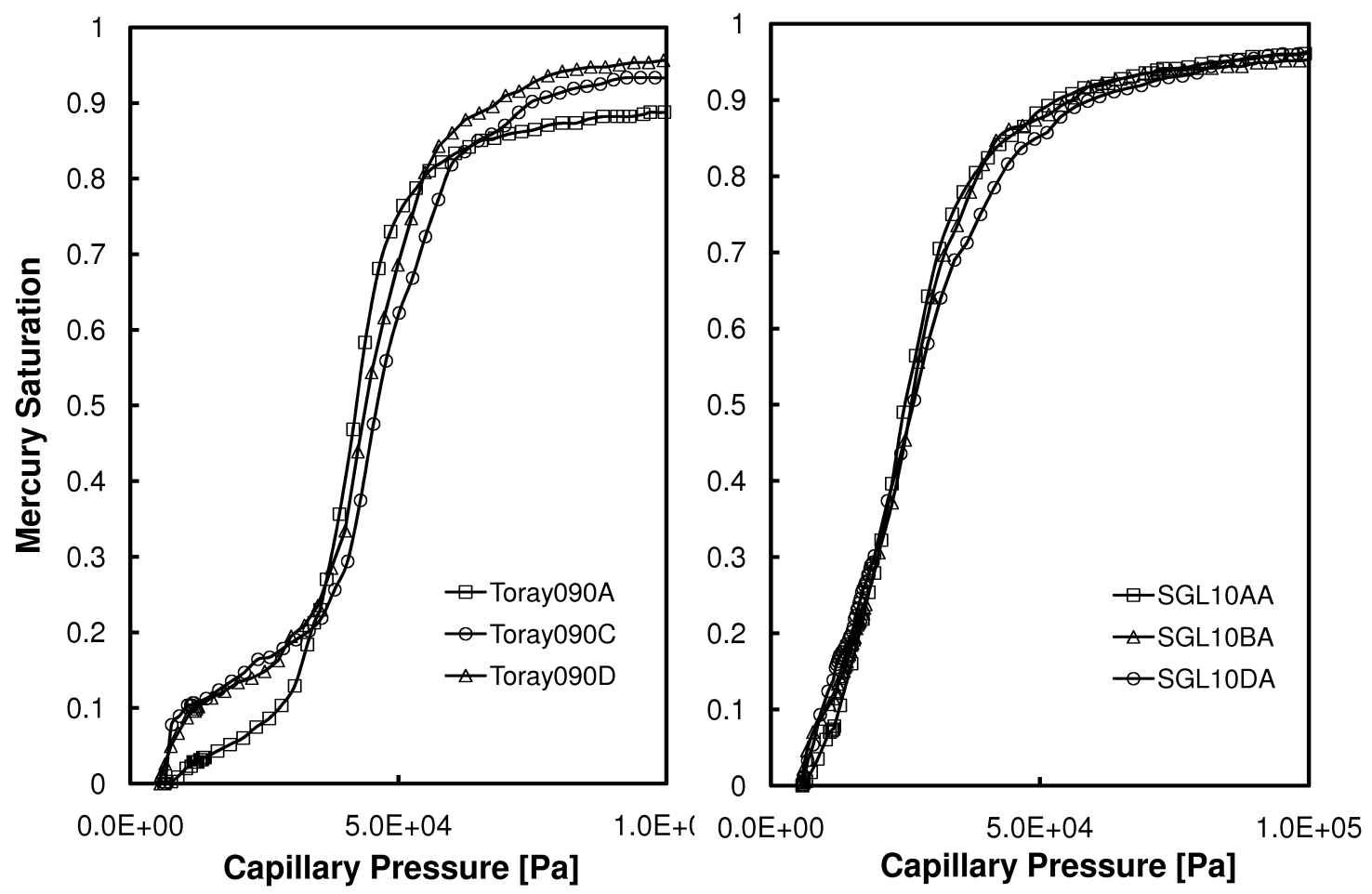

Figure 4: Mercury-air capillary pressure curves for samples with varying PTFE loadings as given in Table 1. Left: Toray 090. Right: SGL10. Data were obtained for pressures up to $400 \mathrm{kPa}$, but are shown only up to $100 \mathrm{kPa}$ for clarity. 

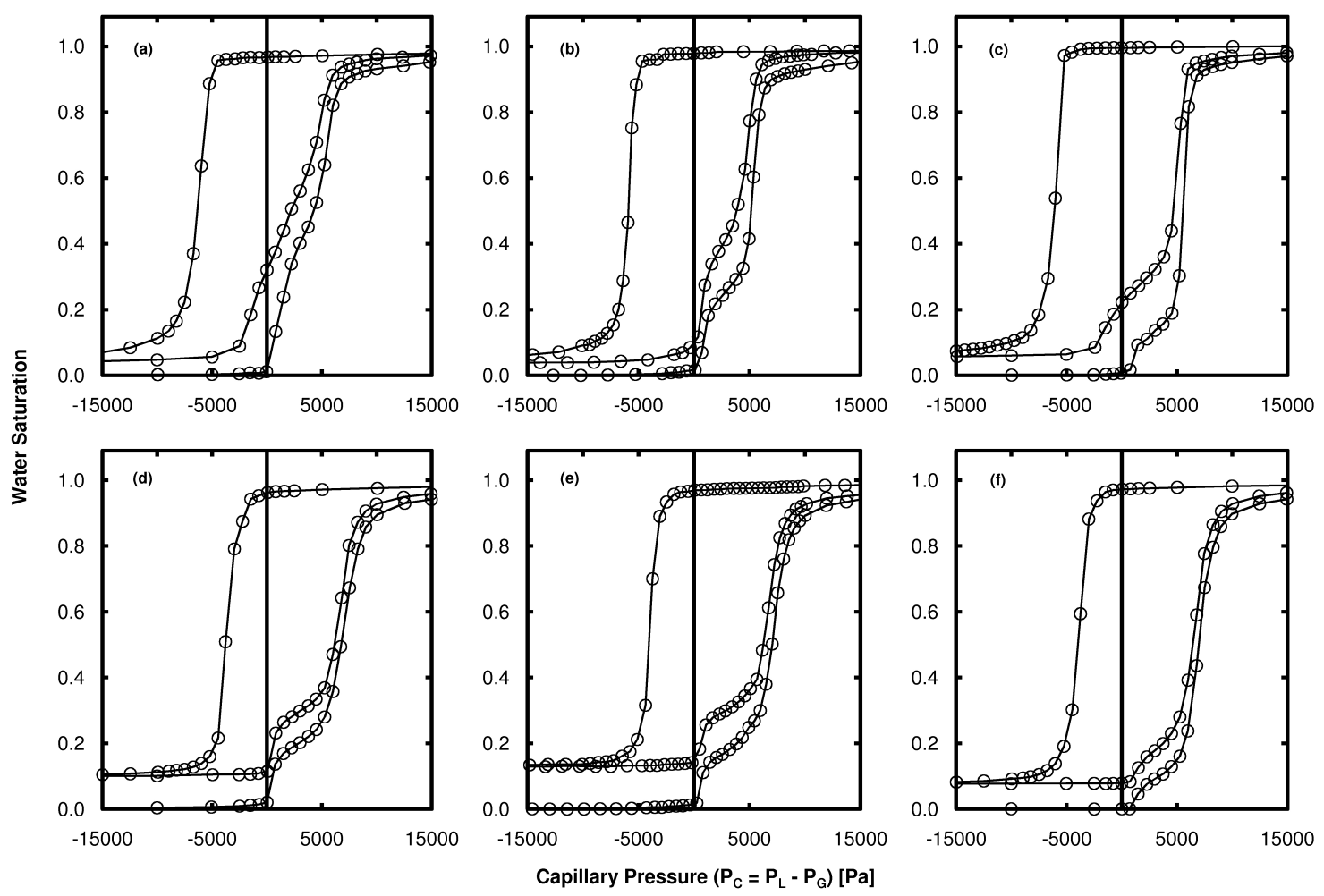

Figure 5: Water-air capillary pressure curves for various Toray samples. (a) Toray 060A. (b) Toray 090A. (c) Toray 120A. (d) Toray 060D. (e) Toray 090D. (f) Toray 120C. Data points were collected for $-30,000 \mathrm{~Pa}<P_{C}<30,000 \mathrm{~Pa}$, but a smaller range is shown for clarity. Physical properties of each material are listed in Table 1. 

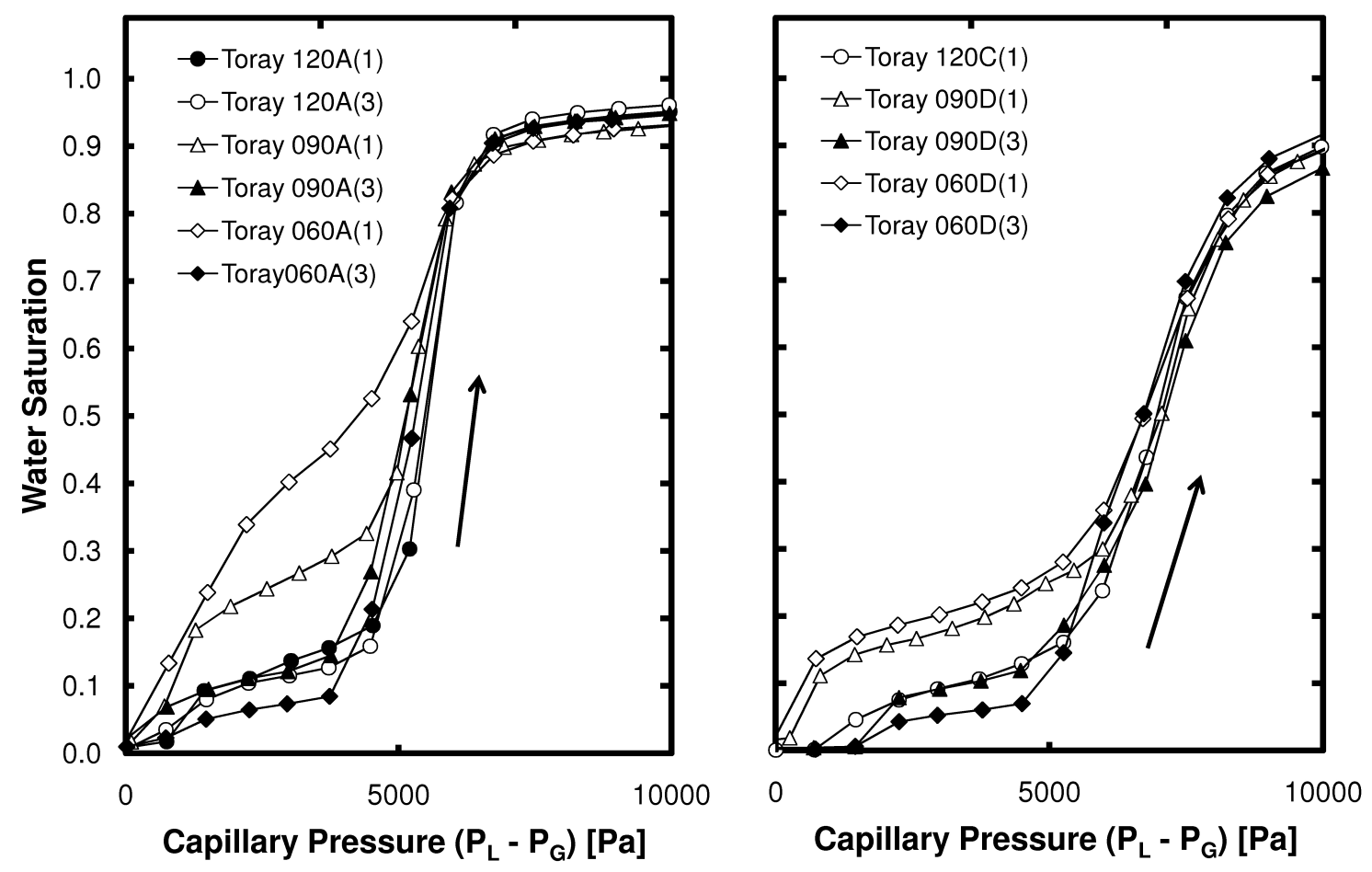

Figure 6: Primary water injection curves (truncated to $10 \mathrm{kPa}$ for clarity) as a function of sample thickness and number of layers (shown in brackets) for Toray samples without PTFE coating (left) and with PTFE coating (right). 


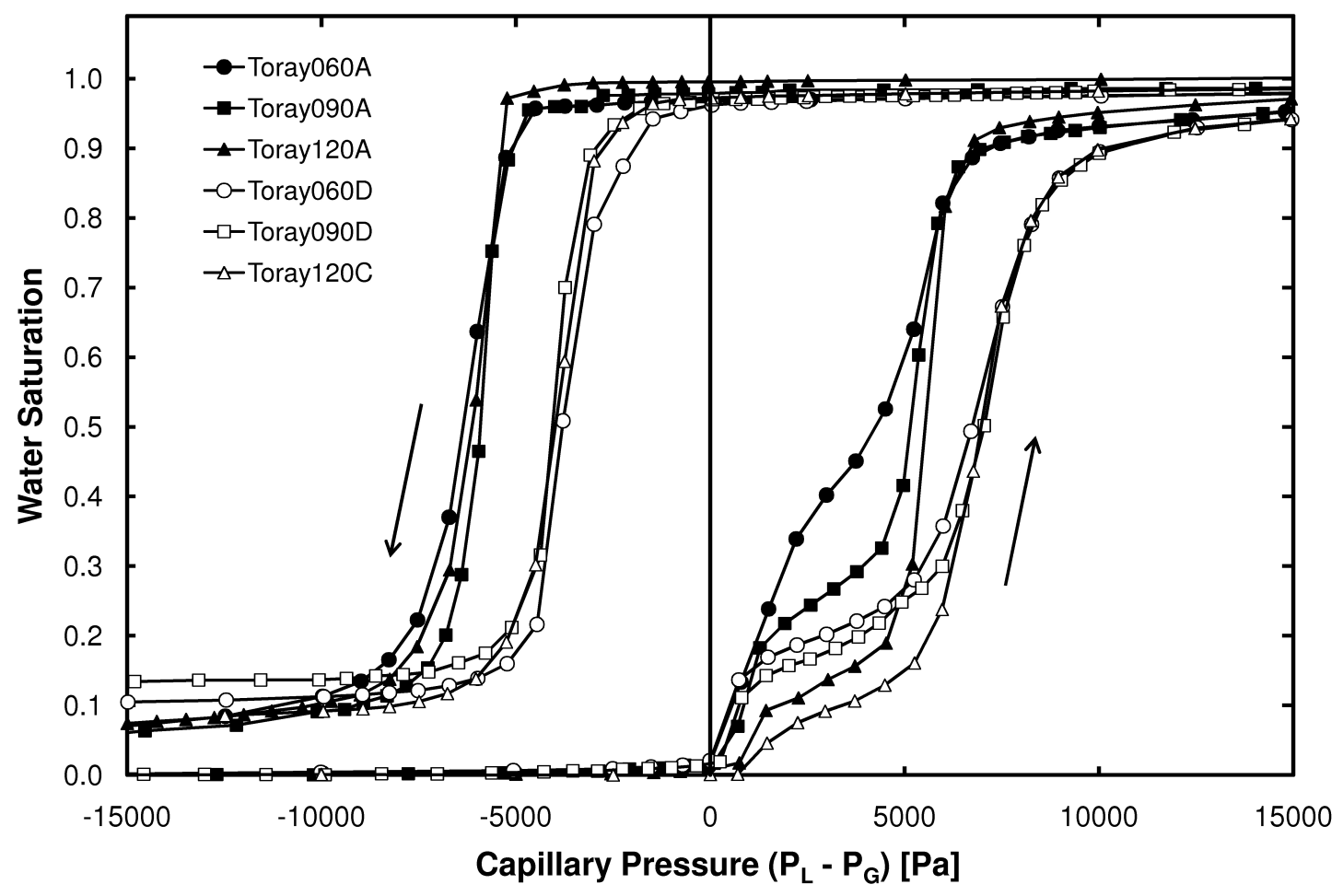

Figure 7: Primary injection and primary withdrawal curves for Toray materials of different thickness with (white markers) and without (black markers) PTFE. Secondary injection curves are not shown for clarity. 


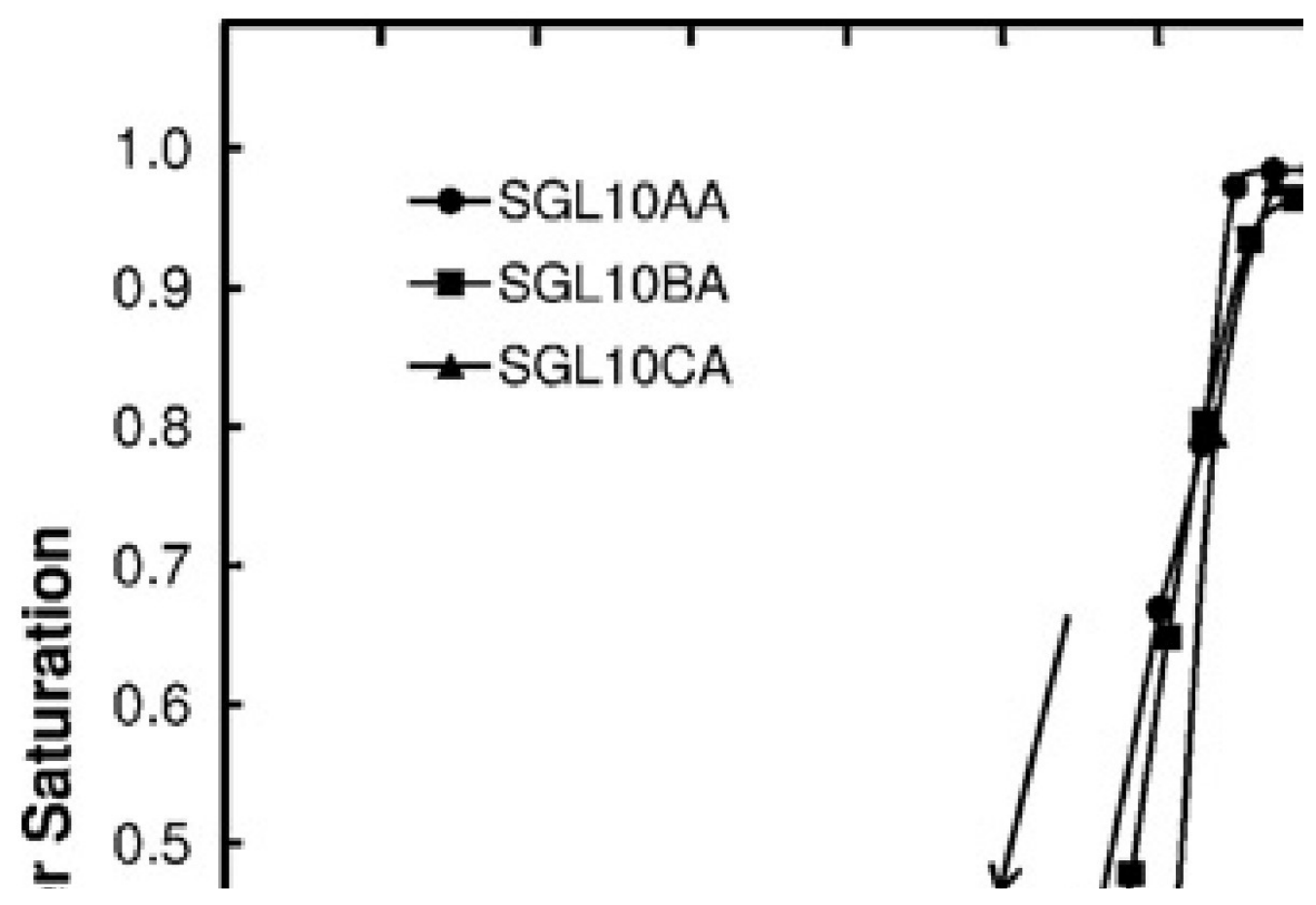

Figure 8: $\quad$ Primary injection and primary withdrawal curves for SGL10 materials with different PTFE loadings. Secondary injection curves are not shown for clarity. 

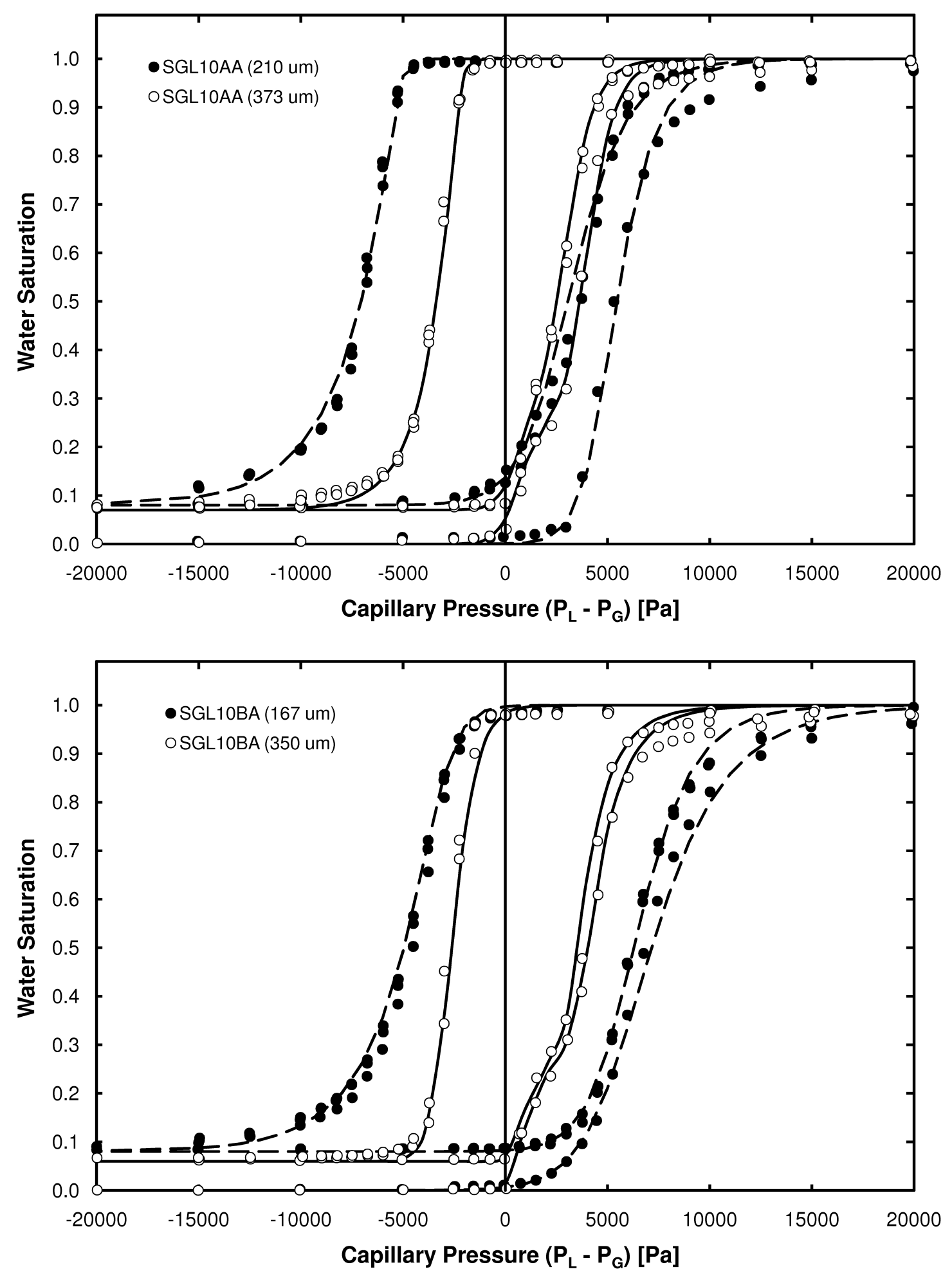

Figure 9: Water-air capillary pressure curves for SGL10AA (top) and SGL10BA (bottom) under compressed (black) and uncompressed (white) conditions. Lines through the data points are fit using the procedure outlined in Appendix A. 

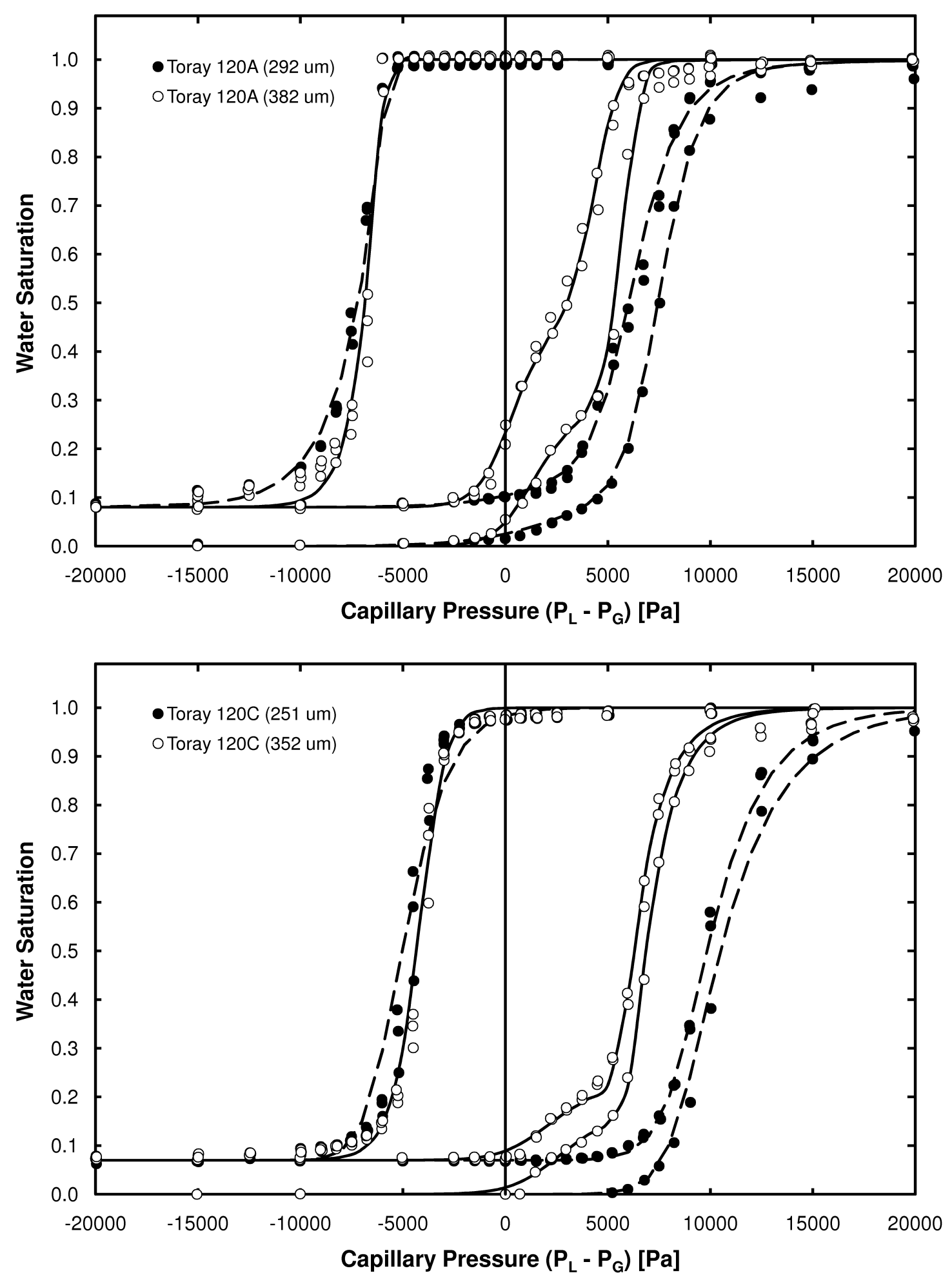

Figure 10: Water-air capillary pressure curves for Toray 120A (top) and Toray 120C (bottom) under compressed (black) and uncompressed (white) conditions. Lines through 
the data points are fit using the procedure outlined in Appendix A.

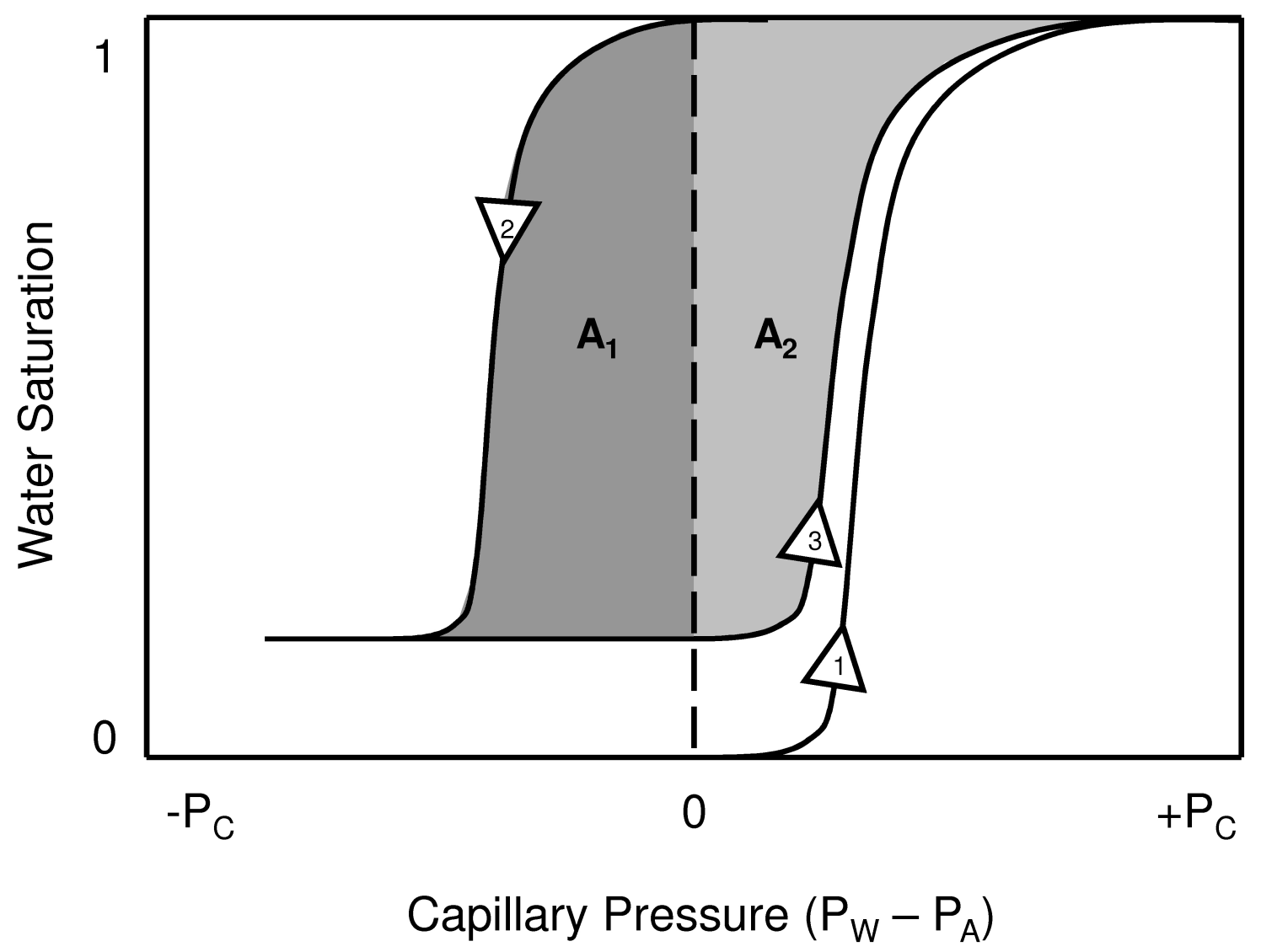

Figure 11: Area definitions used to calculate the US Bureau of Mines wettability index 


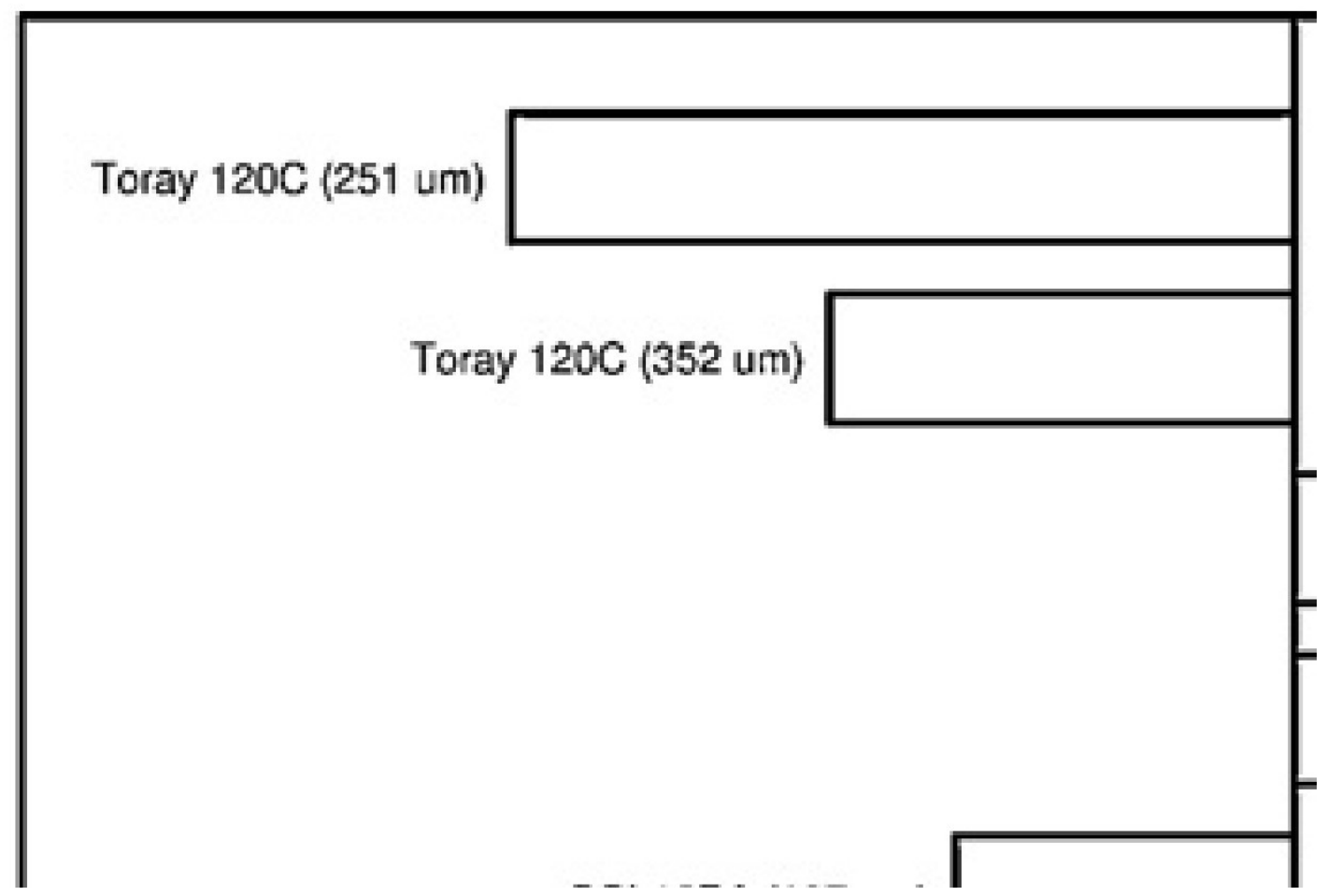

Figure 12: USBM wettability index values for all samples tested under compressed and uncompressed conditions. 